\title{
The Generation of Rossby Waves and Wake Eddies by Small Islands
}

\author{
by R. C. Musgrave ${ }^{1}$, G. Flierl' ${ }^{2}$, and T. Peacock ${ }^{2}$
}

\begin{abstract}
The influence of small islands on zonal geostrophic currents is examined in a two-layer configuration. An analytic solution for steady quasigeostrophic flow is derived under the assumption of no upstream influence and is validated numerically in a time-dependent quasigeostrophic model. Under these conditions solutions are the sum of two eigenmodes, which are either arrested Rossby waves or evanescent depending on background flow conditions (layer speeds, stratification, and latitude). In contrast to homogeneous flows, arrested Rossby waves in two layers can occur even when the depth mean flow is westward and can be generated both to the east and west of the island. A third blocking mode may play a role in general, altering the meridional structure of the zonal flow upstream and downstream of the island. The influence of the quasigeostrophic modes on submesoscale island wake eddies is considered in a two-layer primitive equation model with no-slip boundary conditions at the island. Wake eddy formation is inhibited in the presence of an arrested Rossby wave, though the overall drag is similar.
\end{abstract}

Keywords: Rossby waves, Islands, Quasi-geostrophy

\section{Introduction}

The interaction of large-scale currents with topographic features gives rise to a range of relatively small-scale phenomena, such as wake eddies and lee waves, redistributing the energy of the wind driven circulation and transferring it towards smaller scales and dissipation. In a study of homogeneous quasigeostrophic eastward flow past a cylindrical island, White (1971) presented analytic solutions demonstrating the generation of arrested Rossby waves in the island's lee. The lengthscale of the arrested wave is

$$
L_{\beta}=\left(\frac{u_{0}}{\beta}\right)^{1 / 2}
$$

where $u_{0}$ is the eastward zonal flow speed and $\beta$ is the meridional variation of the Coriolis parameter. A similar result was obtained for homogeneous flows past islands in a channel

1. Woods Hole Oceanographic Institution, Woods Hole, Massachusetts, USA

2. Massachusetts Institute of Technology, Cambridge, Massachusetts, USA

(C) 2018 R. C. Musgrave, G. Flierl, and T. Peacock. 
(Pedlosky and Spall 2015), where the meridional boundaries impose a maximum lengthscale (and consequently phase speed) of the barotropic wave, enabling a supercritical transition when flow speeds become large. In general, arrested waves have significant amplitude for islands with scales that match that of the arrested wave, with relevance for typical oceanic parameters at island scales of approximately $100 \mathrm{~km}$ or more, much smaller than planetary scales. The westward phase propagation of the barotropic Rossby wave is precisely matched by the zonal velocity of the eastward current, causing them to have zero frequency with respect to the island. In addition to setting the downstream flow, these waves influence the frictional boundary layer by generating an asymmetry in the pressure distribution around the island, which inhibits the separation of the flow downstream (Merkine 1980). In doing so, arrested Rossby waves in the lee of islands reduce the propensity of a flow to form submesoscale island wake eddies at fixed Reynolds number (Boyer and Davies 1982). By determining the momentum and energy pathways associated with flow past topography, these processes affect dynamics on both large, quasigeostrophic scales and smaller submesoscales close to the island.

In addition to Rossby waves, blocking modes can form both upstream and downstream of islands leading to zonal jets (Rhines 2007). Though these modes are not necessary to complete a steady solution, they are generally relevant in the ocean, where the timedependent adjustment of a flow may generate blocking structures that extend zonally with time. Zonal jets can also be formed by time-dependent, breaking Rossby waves due to the convergence of Reynolds stresses (Tansley and Marshall 2001).

The role of stratification in these problems has been relatively less studied. The interaction of two-layer quasigeostrophic flow with a small seamount was considered by McCartney (1975) (i.e., topographies having a height much less than the lower layer thickness). Analytic solutions are formed from the two-layer quasigeostrophic eigenmodes and exhibit both arrested Rossby waves and Taylor Columns under certain flow conditions.

In this paper we extend existing single-layer quasigeostrophic theory for flow past islands to two layers. Our solutions use the same eigenmodes of McCartney (1975), each of which can be wavelike or evanescent; however, the island boundary conditions impose a distinct flow structure compared with McCartney's seamount solutions (Section 2). General behaviors across oceanic parameter regimes are discussed in Section 3, demonstrating transitions from wavelike to evanescent behaviour in each mode and supercritical behavior. Specific solutions are considered and compared with a time-dependent quasigeostrophic numerical model in Section 4, where we illustrate the formation of upstream and downstream blocking and the generation of Rossby waves to the west and east of the island under certain conditions. Finally, in Section 5, the influence of the quasigeostrophic outer solution on the formation of submesoscale wake eddies is considered in a two-layer primitive equation simulation, showing the effect of arrested Rossby waves on the boundary layer. We discuss the wave drag associated with the inviscid solution and the form drag from vortex shedding associated with the viscous primitive equation model, demonstrating that vortex shedding dominates drag behavior. We summarize our results in Section 6. 


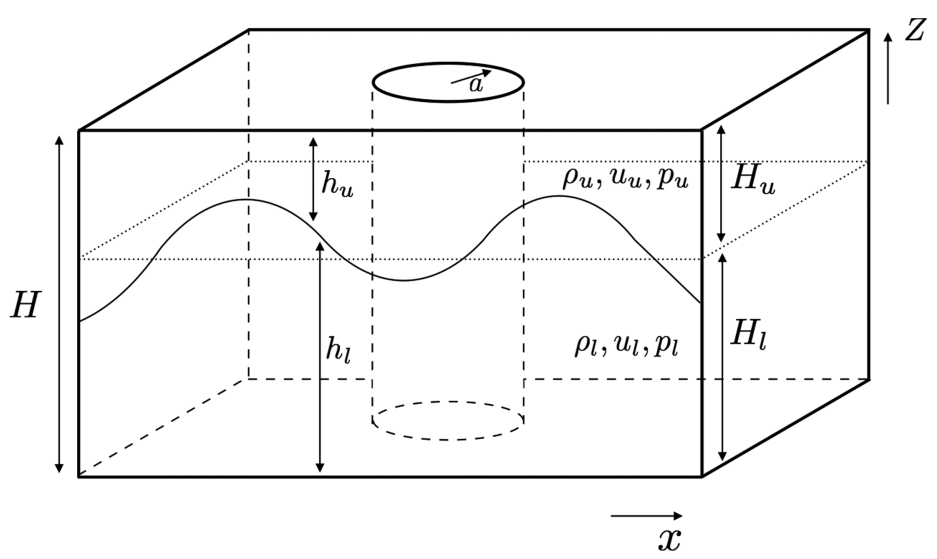

Figure 1. Two layer model configuration: a cylindrical island of radius $a$ is immersed in two layers of density $\rho_{u}, \rho_{l}$.

\section{Quasigeostrophic 2-layer zonal flow past an island}

\section{a. Governing equations}

We consider the steady interaction of a quasigeostrophic, inviscid two-layer shallow water system with a small island (Fig. 1). In the absence of forcing and dissipation, the quasigeostrophic potential vorticity (PV) is conserved in each layer:

$$
\frac{D q_{u}}{D t}=0, \quad \text { and } \quad \frac{D q_{l}}{D t}=0
$$

where we use subscripts $u$ and $l$ to define the upper and lower layers, respectively. The potential vorticities are defined as follows (see, e.g., Salmon 1998):

$$
\begin{aligned}
& q_{u}=\nabla^{2} \psi_{u}+\beta y-\delta \frac{\psi_{u}-\psi_{l}}{R_{D}^{2}}, \\
& q_{l}=\nabla^{2} \psi_{l}+\beta y+(1-\delta) \frac{\psi_{u}-\psi_{l}}{R_{D}^{2}},
\end{aligned}
$$

where the geostrophic streamfunction, $\psi$, is defined with respect to the following geostrophic velocities, $\left(u_{g}, v_{g}\right)=\left(-\psi_{y}, \psi_{x}\right)$. The total depth of the fluid is $H$, with $H_{l}$ and $H_{u}$ the undisturbed height of each layer, and $\delta=H_{l} / H$. The Coriolis parameter is approximated by a linear expression, $f=f_{0}+\beta y$, where $f_{0}$ is the central latitude of the model, and $\beta$ defines its meridional rate of change. The baroclinic deformation radius for the two layers is

$$
R_{D}^{2}=\frac{g^{\prime}}{f_{0}^{2}} \frac{H_{l} H_{u}}{H_{l}+H_{u}}=\frac{g^{\prime} H}{f_{0}^{2}}[\delta(1-\delta)] .
$$


In steady state, the governing equations in each layer (Eq. 2) can be expressed in along$(\zeta)$ and across- $(\psi)$ streamline coordinates,

$$
\frac{\partial(\psi, q)}{\partial(x, y)}=\frac{\partial(\psi, \zeta)}{\partial(x, y)} \frac{\partial(\psi, q)}{\partial(\psi, \zeta)}=\frac{\partial(\psi, \zeta)}{\partial(x, y)} q_{\zeta}=0 .
$$

The coordinate transformation becomes singular at stagnation points, where the geometric Jacobian term, $\frac{\partial(\psi, \zeta)}{\partial(x, y)}$, is zero. Away from such points, the Jacobian is nonzero, and the governing equations are concisely expressed

$$
q_{\zeta}=0
$$

explicitly demonstrating that the potential vorticity in each layer is conserved along streamlines.

One modal solution to Eq. (5) is readily apparent: for a strictly zonal flow, Eq. (5) can be written $q_{x}=0$, with wavelike solutions having $k=0$. This "blocking" mode can lead to adjustments to the zonal flow far upstream and downstream of the island (Rhines 2007). Setting the amplitude and structure of this mode has certain arbitrary aspects and amounts to specifying the upstream flow conditions for a steady solution at the island. In any realistic time-dependent problem, blocking modes will set the upstream zonal flow during the establishment of the steady solution at the island: though artificial, the radiation of blocking modes could adjust an initially nonuniform zonal flow to a uniform steady state; alternatively, an initially uniform zonal flow could adjust to a nonuniform steady state. In this section we specify the upstream flow to be zonally uniform without considering the time-dependent problem by which that condition arose. More general solutions with nonuniform upstream conditions set by radiating blocking modes will be discussed further in Sections 3 and 4, where we illustrate the process in time-dependent numerical solutions.

We define a background streamfunction $(\Psi)$ and $\mathrm{PV}(Q)$ in each layer associated with a uniform zonal flow and seek solutions for perturbations from the background, with $\psi=$ $\Psi+\phi$ and $q=Q+q^{\prime}$ :

$$
\begin{aligned}
\Psi_{u}=-u_{u} y \quad \text { and } \quad \Psi_{l} & =-u_{l} y, \\
Q_{u}=-\frac{\beta \Psi_{u}}{u_{u}}+\delta \frac{\left(u_{u}-u_{l}\right) y}{R_{D}^{2}} \quad \text { and } \quad Q_{l} & =-\frac{\beta \Psi_{l}}{u_{l}}-(1-\delta) \frac{\left(u_{u}-u_{l}\right) y}{R_{D}^{2}}, \\
q_{u}^{\prime}=\nabla^{2} \phi_{u}-\delta \frac{\phi_{u}-\phi_{l}}{R_{D}^{2}} & \text { and } \quad q_{l}^{\prime}=\nabla^{2} \phi_{l}+(1-\delta) \frac{\phi_{u}-\phi_{l}}{R_{D}^{2}}
\end{aligned}
$$

The solution proceeds by integrating Eq. (5) in each layer in the along-streamline direction. The integration is performed from a point far upstream of the island where the zonal flow is uniform and $\phi=0$. The upstream potential vorticity is then simply prescribed by the background potential vorticity of the flow. In addition to the previously discussed blocking modes, we anticipate two further modal solutions to arise in this two-layer flow, each 
of which can have group velocities in either zonal direction. By prescribing the potential vorticity upstream we limit our solutions in this section to situations where, on one side of the island, both modes decay with distance so that their potential vorticity perturbations tend to zero. Parameter regimes where this is not expected to be the case will be discussed in Section 3, and an explicit solution is illustrated numerically in Section 4.

By matching potential vorticity at the undisturbed upstream to that at the island along a streamline, the coupled equations in each layer become the following:

Upper layer

$$
\nabla_{h}^{2} \phi_{u}-\left(\frac{\delta}{R_{D}^{2}} \frac{u_{l}}{u_{u}}-\frac{\beta}{u_{u}}\right) \phi_{u}=-\frac{\delta}{R_{D}^{2}} \phi_{l}
$$

Lower layer

$$
\nabla_{h}^{2} \phi_{l}-\left(\frac{1-\delta}{R_{D}^{2}} \frac{u_{u}}{u_{l}}-\frac{\beta}{u_{l}}\right) \phi_{l}=-\frac{1-\delta}{R_{D}^{2}} \phi_{u} .
$$

These integrated equations are linear because $q$ is a linear function of $\psi$ in each layer; however, solutions also satisfy the nonlinear problem exactly. Solutions are not stable if the meridional gradient of the background potential vorticity, $Q_{y}$, changes sign between layers. We define the following constants:

$$
\begin{gathered}
F_{l}=\frac{1-\delta}{R_{D}^{2}}, \quad F_{u}=\frac{\delta}{R_{D}^{2}} \\
\alpha_{l}=-\frac{\beta}{u_{l}}, \quad \alpha_{u}=-\frac{\beta}{u_{u}} \\
N_{u}=\alpha_{u}+F_{u} \frac{u_{l}}{u_{u}}, \quad N_{l}=\alpha_{l}+F_{l} \frac{u_{u}}{u_{l}}, \quad N=F_{u} F_{l} .
\end{gathered}
$$

Combining layer Eqs. (7) and (8) lead to linear fourth-order equations in each layer:

$$
\left[\nabla^{2}+k_{A}^{2}\right]\left[\nabla^{2}+k_{B}^{2}\right] \phi_{u}=0 \text { and }\left[\nabla^{2}+k_{A}^{2}\right]\left[\nabla^{2}+k_{B}^{2}\right] \phi_{l}=0,
$$

where

$$
\begin{aligned}
k_{A}^{2} & =-\frac{1}{2}\left(N_{l}+N_{u}\right)+\frac{1}{2}\left[\left(N_{l}+N_{u}\right)^{2}-4\left(N_{u} N_{l}-N\right)\right]^{1 / 2}, \\
k_{B}^{2} & =-\frac{1}{2}\left(N_{l}+N_{u}\right)-\frac{1}{2}\left[\left(N_{l}+N_{u}\right)^{2}-4\left(N_{u} N_{l}-N\right)\right]^{1 / 2}
\end{aligned}
$$

represent the two eigenmode solutions. Using $\nabla^{2} \rightarrow k_{A}^{2}, k_{B}^{2}$ in Eqs. (7) and (8), the relative amplitudes of the perturbation streamfunction, $\phi$, in the lower to upper layer for each mode, $\Delta_{A}$ and $\Delta_{B}$ are

$$
\Delta_{A}=\frac{F_{u} \frac{u_{l}}{u_{u}}+\alpha_{u}+k_{A}^{2}}{F_{u}} \text { and } \Delta_{B}=\frac{F_{u} \frac{u_{l}}{u_{u}}+\alpha_{u}+k_{B}^{2}}{F_{u}}
$$


As we are considering a circular island it is natural to work with general solutions to Eqs. (9) in polar coordinates so we can readily apply the boundary condition at $r=a$, the radius of the island. Solutions in each of the layers are sines/cosines in $\theta$, and Bessel functions in $r: X_{1 n}, X_{2 n}$, which are either $J_{n}, Y_{n}$ (radiating) or $I_{n}, K_{n}$ (evanescent) depending on the sign of $k^{2}$. The full solution is a sum of both eigenmodes:

$$
\begin{aligned}
{\left[\begin{array}{c}
\phi_{u} \\
\phi_{l}
\end{array}\right]=} & {\left[\begin{array}{c}
1 \\
\Delta_{A}
\end{array}\right]\left(\left[a_{A n} \cos n \theta+b_{A n} \sin n \theta\right] X_{1 n}\left(k_{A} r\right)+\left[c_{A n} \cos n \theta+d_{A n} \sin n \theta\right] X_{2 n}\left(k_{A} r\right)\right) } \\
& +\left[\begin{array}{c}
1 \\
\Delta_{B}
\end{array}\right]\left(\left[a_{B n} \cos n \theta+b_{B n} \sin n \theta\right] X_{1 n}\left(k_{B} r\right)+\left[c_{B n} \cos n \theta+d_{B n} \sin n \theta\right] X_{2 n}\left(k_{B} r\right)\right) .
\end{aligned}
$$

Constants $a_{A n}, b_{A n} \ldots$ etc. are determined by the boundary conditions. Depending on the signs of $k_{A}^{2}, k_{B}^{2}$, either both modes are wavelike, both modes are evanescent, or one mode is wavelike and one mode is evanescent.

\section{b. Boundary conditions}

We impose that there is no flow into the island boundary at each order of the quasigeostrophic approximation.

$$
\begin{aligned}
& \mathbf{u}^{(0)} \cdot \vec{n}=0 \\
& \mathbf{u}^{(1)} \cdot \vec{n}=0,
\end{aligned}
$$

At zeroth order the flow is geostrophic, and the boundary condition is equivalent to

$$
\left.\hat{t} \cdot \nabla \psi\right|_{C_{I}}=0
$$

implying that the geostrophic streamfunction, $\psi$, is constant on the boundary of the island in each layer and has no contributions for $n>0$. The constant value that the streamline takes on the island is determined by the boundary condition on the order Rossby number (ageostrophic) flow; for this, we consider the influence of a linear drag at the boundary, $r \mathbf{u}^{(0)}$, in the first order quasigeostrophic momentum equation. At steady state, an integral of the momentum balance along the boundary of the island becomes

$$
r \oint_{C_{I}} \mathbf{u}^{0} \cdot \hat{t}=0
$$

where $C_{I}$ is the island boundary. The circulation around the island is defined by $\Gamma=$ $\oint_{C_{I}} \mathbf{u}^{0} \cdot \hat{t}$, and so this condition suggests that a physically reasonable condition is that there is no net circulation around the island, and the flow is meridionally symmetric. The value of the streamfunction at the island (the Island streamfunction, $\psi_{I}$ ) consistent with this condition is that $\psi_{I}=0$. 


\section{c. Solutions}

The zero normal flow boundary conditions are sufficient to fully determine the unknowns in Eq. (12) when solutions are evanescent (i.e., $k^{2}<0$ ), as we can discard the Bessel function that grows with distance from the island. When the solutions are wavelike, however, we impose the condition of no upstream influence by the island, i.e., for $\theta=\pi$ or 0 (depending on which side is upstream) as $r \rightarrow \infty, \partial_{\theta} \phi=0$.

The full solution is a sum of both modes, each of which can be wavelike or evanescent. We summarize below the three possible combinations.

i. Both mode evanescent $\left(k_{A}^{2}, k_{B}^{2}<0\right)$ : Solutions are modified Bessel functions $\left(X_{1 n}, X_{2 n} \rightarrow\right.$ $I_{n}, K_{n}$ ), and we discard the $I_{n}$ 's on energetic grounds:

$$
\left[\begin{array}{c}
\phi_{u} \\
\phi_{l}
\end{array}\right]=\left[\begin{array}{c}
1 \\
\Delta_{A}
\end{array}\right]\left[c_{A n} \cos n \theta+d_{A n} \sin n \theta\right] K_{n}\left(k_{A} r\right)+\left[\begin{array}{c}
1 \\
\Delta_{B}
\end{array}\right]\left[c_{B n} \cos n \theta+d_{B n} \sin n \theta\right] K_{n}\left(k_{B} r\right)
$$

Upon application of the boundary condition that $\psi_{I}=\phi_{I}(r=a)-u a \sin \theta=0$ in each layer, only the odd (sine) Bessel function modes survive for $n=1$, and the solution is written

$$
\left[\begin{array}{c}
\psi_{u} \\
\psi_{l}
\end{array}\right]=\left(\left[\begin{array}{c}
1 \\
\Delta_{A}
\end{array}\right] \frac{-u_{u} a \Delta_{B}+u_{l} a}{K_{1}\left(k_{A} a\right)\left(\Delta_{A}-\Delta_{B}\right)} K_{1}\left(k_{A} r\right)+\left[\begin{array}{c}
1 \\
\Delta_{B}
\end{array}\right] \frac{u_{u} a \Delta_{A}-u_{l} a}{K_{1}\left(k_{B} a\right)\left(\Delta_{A}-\Delta_{B}\right)} K_{1}\left(k_{B} r\right)-\left[\begin{array}{l}
u_{u} \\
u_{l}
\end{array}\right] r\right) \sin \theta .
$$

where $\Delta_{A}$ and $\Delta_{B}$ are defined in Eq. (11).

ii. Both mode wave-like $\left(k_{A}^{2}, k_{B}^{2}>0\right)$ : The general solution in this case is

$$
\begin{aligned}
{\left[\begin{array}{c}
\phi_{u} \\
\phi_{l}
\end{array}\right]=} & {\left[\begin{array}{c}
1 \\
\Delta_{A}
\end{array}\right]\left(\left[a_{A n} \cos n \theta+b_{A n} \sin n \theta\right] J_{n}\left(k_{A} r\right)+\left[c_{A n} \cos n \theta+d_{A n} \sin n \theta\right] Y_{n}\left(k_{A} r\right)\right) } \\
& +\left[\begin{array}{c}
1 \\
\Delta_{B}
\end{array}\right]\left(\left[a_{B n} \cos n \theta+b_{B n} \sin n \theta\right] J_{n}\left(k_{B} r\right)+\left[c_{B n} \cos n \theta+d_{B n} \sin n \theta\right] Y_{n}\left(k_{B} r\right)\right) .
\end{aligned}
$$

As in the evanescent case, the requirement that $\psi_{I}=0$ enforces that the coefficients of the cosine Bessel functions are zero: $c_{A n}=c_{B n}=a_{A n}=a_{B n}=0$. To determine the remaining coefficients, we adopt the approximate method used by White (1971), which makes the waves vanish upstream in the vicinity of the $y$-axis by setting $d \psi / d \theta=0$ at $\theta=\pi$ (or $-\pi$ in the case when the waves are to the west of the island). Thus, we are not exactly satisfying the condition of vanishing influx of wave energy over the western (eastern) half plane. Alternatives, such as expanding in the "cylindrical lee wave functions" of Miles (1968), satisfy the flux condition but, when truncated, do not precisely satisfy the conditions at the island. Solving the linear problem with damping or Laplace transforms of 
an initial state leads to complicated inversions. We expect our solutions will closely resemble these and show in the next section that they remain steady when realized numerically. The solution method and constants are detailed in Appendix A, and here we simply present the solution where the upstream condition is applied to the west of the island:

$$
\begin{aligned}
{\left[\begin{array}{l}
\psi_{u} \\
\psi_{l}
\end{array}\right]=} & {\left[\begin{array}{c}
1 \\
\Delta_{A}
\end{array}\right]\left(\left[b_{A 1} J_{1}\left(k_{A} r\right)+d_{A 1} Y_{1}\left(k_{A} r\right)\right] \sin \theta+\left[b_{A 2} J_{2}\left(k_{A} r\right)+d_{A 2} Y_{2}\left(k_{A} r\right)\right] \sin 2 \theta\right) } \\
& +\left[\begin{array}{c}
1 \\
\Delta_{B}
\end{array}\right]\left(\left[b_{B 1} J_{1}\left(k_{B} r\right)+d_{B 1} Y_{1}\left(k_{B} r\right)\right] \sin \theta+\left[b_{B 2} J_{2}\left(k_{B} r\right)+d_{B 2} Y_{2}\left(k_{B} r\right)\right] \sin 2 \theta\right) \\
& -\left[\begin{array}{c}
u_{u} \\
u_{l}
\end{array}\right] r \sin \theta
\end{aligned}
$$

Note the presence of the $\sin 2 \theta$ term, associated with the second Bessel harmonic, which was not present in the evanescent solution. This term makes the flow past the island zonally asymmetric - accelerating tangential flow in the lee of the island-with important implications for both the separation of a frictional boundary layer (see Section 5) and form drag on the island.

iii. Mixed mode evanescent/wave-like: These occur when $k_{A}^{2}>0, k_{B}^{2}<0$, or vice versa. We write the wavelike eigenvalue $k_{W}$ and the evanescent eigenvalue $k_{E}$, in which case the general solution is

$$
\begin{aligned}
{\left[\begin{array}{c}
\phi_{u} \\
\phi_{l}
\end{array}\right]=} & {\left[\begin{array}{c}
1 \\
\Delta_{W}
\end{array}\right]\left(\left[b_{W n} \sin n \theta\right] J_{n}\left(k_{W} r\right)+\left[d_{W n} \sin n \theta\right] Y_{n}\left(k_{W} r\right)\right) } \\
& +\left[\begin{array}{c}
1 \\
\Delta_{E}
\end{array}\right]\left[d_{E n} \sin n \theta\right] K_{n}\left(k_{E} r\right) .
\end{aligned}
$$

The constants are derived in Appendix B, in which upstream is taken to be to the west of the island. The solution is

$$
\begin{aligned}
{\left[\begin{array}{c}
\phi_{u} \\
\phi_{l}
\end{array}\right]=} & {\left[\begin{array}{c}
1 \\
\Delta_{W}
\end{array}\right]\left(\left[b_{W 1} J_{1}\left(k_{W} r\right)+d_{W 1} Y_{1}\left(k_{W} r\right)\right] \sin \theta+\left[b_{W 2} J_{2}\left(k_{W} r\right)+d_{W 2} Y_{2}\left(k_{W} r\right)\right] \sin (2 \theta)\right) } \\
& +\left[\begin{array}{c}
1 \\
\Delta_{E}
\end{array}\right] d_{E 1} K_{1}\left(k_{E} r\right) \sin \theta
\end{aligned}
$$

\section{General behaviors}

The steady behavior of the quasigesotrophic response to an island in a two-layer flow is determined by the sum of two modal solutions, which can each be either wavelike or evanescent depending on background flow conditions. These eigenmodes form the basis for linear solutions in two-layer quasigeostrophic flows and were used by McCartney (1975) to study 
flow over small seamounts where arrested Rossby waves and Taylor columns can occur. Our discussion in this section expands upon that in McCartney (1975), and we examine the general behavior of arrested solutions in two-layer flows for varying layer speeds for given latitudes and stratifications. The generation of these modal solutions by the adjustment of a geostrophic flow around an island will be illustrated with specific examples in Section 4.

Properties of each mode are presented in Figure $2\left(R_{D}=106 \mathrm{~km}\right)$ and Figure $3\left(R_{D}=43\right.$ $\mathrm{km}$ ), which can be thought of as regions with the same stratification but at two different latitudes. We have grouped modes A and B from Section 2 according to the relative sign of the streamfunction perturbation in the upper and lower layers, $\Delta$ (defined in Eq. 11). When $\Delta>0$, perturbations in each layer have the same sign and we call the mode "barotropiclike" (subscript " $T$ "). Conversely, when $\Delta<0$ perturbations have opposite sign and the mode is "baroclinic-like," subscript "C" (Figs. 2; 3c, d). The barotropic-like mode is strictly barotropic only where $\Delta=1$ (i.e., no vertical shear), and the baroclinic-like mode is strictly baroclinic only where $\Delta=-H_{u} / H_{l}$ (i.e., the transports in each layer are equal and opposite). These "pure mode" regimes are indicated by solid black contours in panels $\mathrm{c}$ and $\mathrm{d}$ and occur when either the background flow is barotropic or when one of the modal wavenumber passes through zero or infinity. For other values of $\Delta$, modes have a mixed behavior, with baroclinic-like modes having nonzero depth integrated transport, and barotropic-like modes having vertical shear. Where each mode has large, opposite signs of $\log \Delta$, the response in each layer is approximately uncoupled, with flows in each layer dominated by a single lengthscale.

The transition between wavelike and evanescent behavior by each mode across a range of upper- and lower-layer flow speeds is shown in panels a and b of Figures 2 and 3. Where $k^{2}>0$, arrested solutions are Rossby waves, indicated by red contours, whereas $k^{2}<0$, indicates evanescent responses. When either both layers are eastward or when the layers have opposite direction, at least one mode is wavelike. When both layers are westward, both modes are evanescent. In the two-layer case, a wavelike mode may occur even when the depth mean speed $\left(u_{b t}=\delta u_{l}+(1-\delta) u_{u}\right)$ is westward, in contrast to the homogeneous result of White (1971).

The transition to wavelike or evanescent behavior occurs when the wavenumber changes sign by either passing through zero or infinity, and these are contoured in black in panels a and $b$. When flows are eastward in both layers, the baroclinic-like mode undergoes a supercritical transition that coincides with pure barotropic and baroclinic behaviors of the modes (indicated by black contours in panels $\mathrm{c}$ and $\mathrm{d}$ ). At this point the baroclinic wave attains infinite wavelength and its fastest phase speed and is demarcated by the line, $u_{b t}=R_{D}^{2} \beta$, indicated by a black contour in Figures $2 \mathrm{~b}$ and $3 \mathrm{~b}$ (see Appendix C). For faster $u_{b t}$, no baroclinic phase speed can match the oncoming flow to form an arrested wave, and the solution at the island is evanescent. Interestingly, the deformation radius at the transition,

$$
R_{D}=\sqrt{\frac{u_{b t}}{\beta}}=L_{\beta}
$$



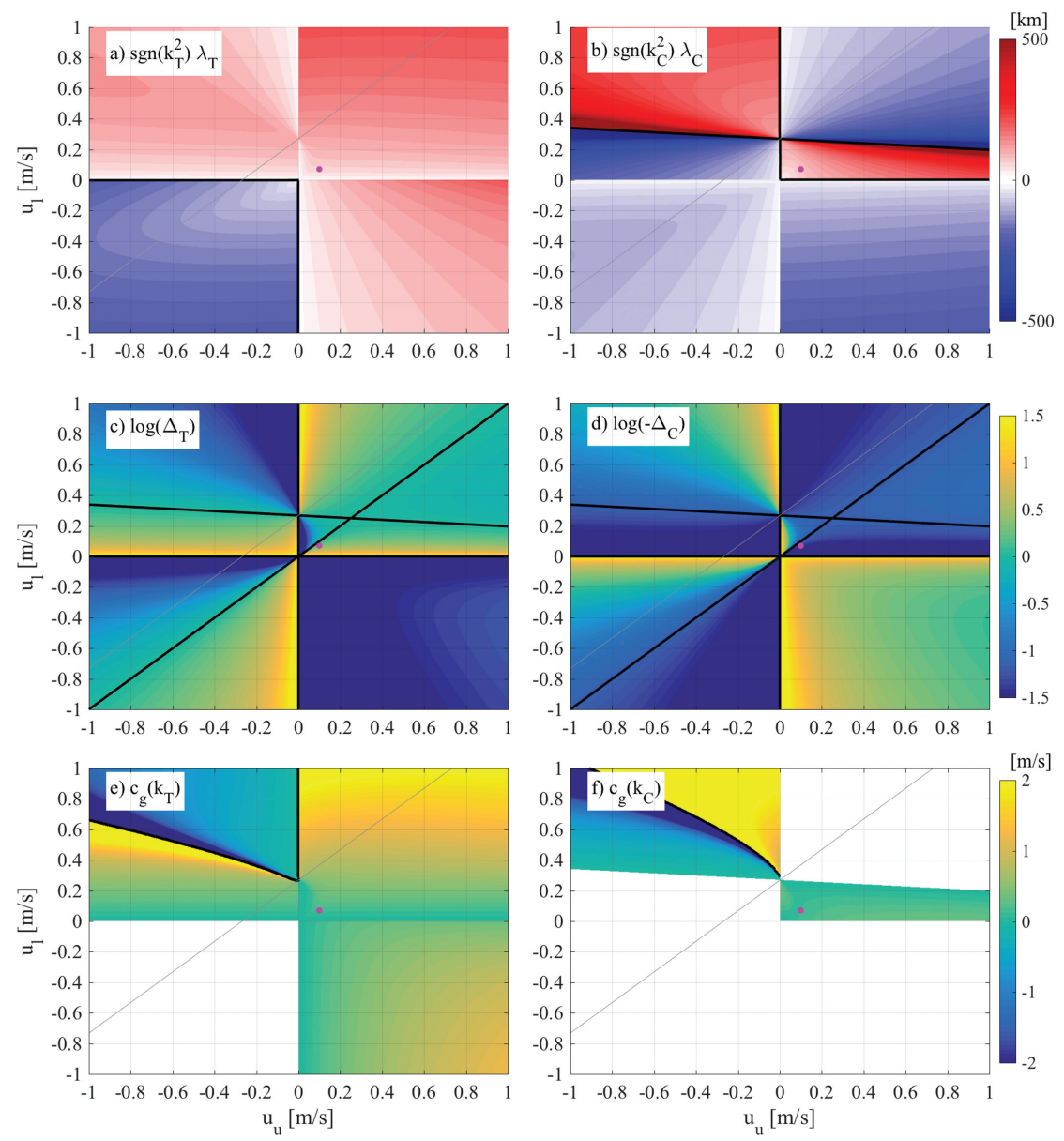

Figure 2. Regime diagrams for $R_{D}=106 \mathrm{~km}, \delta=0.93$. Modes separated by $\operatorname{sgn}(\Delta)$ into barotropiclike (left column, underscore " $T$ ") and baroclinic-like (right column, underscore " $C$ "). Upper panels $(a, b)$ : wavelength (magnitude of color) and nature of response (sign of color: wavelike - red; evanescent - blue) for each mode. Middle panels (c, d): $\log (\Delta)$ for each mode, pure barotropic and baroclinic behavior along black contours. Lower panels $(\mathrm{e}, \mathrm{f})$ : zonal group velocity plane wave with $k=k_{T}, k_{C} ; l=0$ for wavelike solutions. Magenta dot indicates parameter of specific solution considered in text. Thin grey contour indicates transition between stable (low shear) and unstable (high shear) background states.

is the lengthscale of arrested waves in homogeneous flows, $L_{\beta}$, and determines the scales of the Rossby wave island wake solutions in White (1971).

Following the behavior of the baroclinic-like mode, higher modes associated with more layers or continuous stratification would become supercritical at even lower values of $u_{b t}$. 

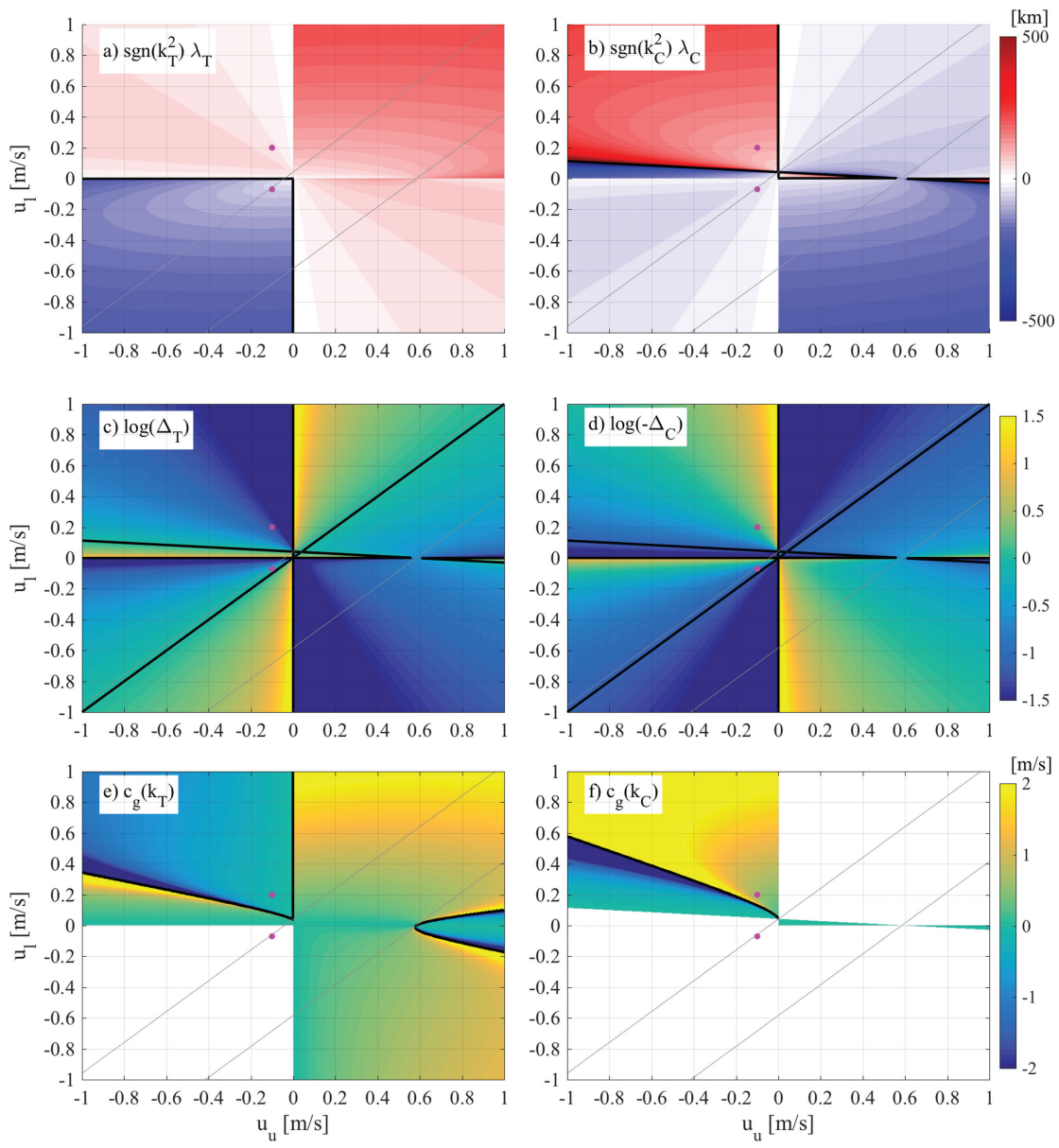

Figure 3. Regime diagrams for $R_{D}=43 \mathrm{~km}, \delta=0.93$. Modes separated by $\operatorname{sgn}(\Delta)$ into barotropiclike (left column, underscore "T") and baroclinic-like (right column, underscore " $C$ "). Upper panels $(\mathrm{a}, \mathrm{b})$ : wavelength (magnitude of color) and nature of response (wavelike: red, evanescent: blue) for each mode. Middle panels $(\mathrm{c}, \mathrm{d}): \log (\Delta)$ for each mode, pure barotropic and baroclinic behavior along black contours. Lower panels (e, f): zonal group velocity plane wave with $k=k_{T}, k_{C} ; l=0$. $c_{g}=0$ is contoured in black. Magenta dots indicate parameters of specific solutions considered in text. Thin grey contours indicate transition between stable (low shear) and unstable (high shear) background states.

As such, we expect that this two-layer model captures the qualitative behavior of island flow for a wide range of oceanic parameters.

For a given latitude and stratification (and, hence, $R_{D}$ ), $L_{\beta}$ increases with barotropic transport. For $L_{\beta}<R_{D}$, eastward flows in both layers arrest two Rossby wave modal solutions, and mixed eastward/westward flows (quadrants 2 and 4) arrest a barotropic-like 
Rossby wave and baroclinic-like evanescent mode. For $L_{\beta}>R_{D}$, the baroclinic-like mode transitions to evanescent for eastward flows in each layer and is wavelike for mixed eastward/westward flows.

The direction of "upstream" of the island is determined by consideration of the group velocity of the wave. The dispersion relation for two-layer plane waves is derived from Eq. (2), linearized around a steady zonal background state and assuming a solution of the form $e^{i(k x+l y-\omega t)}$, where $k, l$ are zonal and meridional wavenumbers, and $\omega$ is the frequency of the wave

$$
\begin{aligned}
& {\left[K^{2}+F_{u}+F_{l}\right] K^{2} \omega^{2}+\left[-K^{2}\left[K^{2}\left(u_{l}+u_{u}\right)+u_{l}\left(F_{u}+N_{l}\right)+u_{u}\left(F_{l}+N_{u}\right)\right]\right.} \\
& \left.\quad+u_{l}\left(F_{l} F_{u}-N_{l} F_{u}\right)+u_{u}\left(F_{u} F_{l}-N_{u} F_{l}\right)\right] k \omega \\
& \quad+u_{u} u_{l}\left[\left(N_{l}+K^{2}\right)\left(N_{u}+K^{2}\right)-N\right] k^{2}=0 .
\end{aligned}
$$

$K^{2}=k^{2}+l^{2}$ is the magnitude of the cartesian wave vector. For $\omega=0, l=0$, the expression reverts to Eq. (10) (though the interpretation of $k$ in that expression is a radial, rather than Cartesian, wavenumber). The group velocity is defined

$$
\overrightarrow{c_{g}}=\left(\frac{\partial \omega}{\partial k}, \frac{\partial \omega}{\partial l}\right)
$$

and an indication of the zonal group velocities of the wavelike modal solutions of Section 2 are estimated by setting $\omega=l=0$, and $k=k_{T}, k_{C}$. These values are contoured for wavelike solutions in the lowest panels (e and f) of Figures 2 and 3 and represent the group velocities of arrested plane waves with infinite meridional scale at these parameters. They are suggestive, rather than predictive, of the group velocities associated with the radial modes in Section 2. For the most part, where solutions are wavelike the group velocity is eastward, indicating that wave wakes form to the east of the island as in a homogeneous flow. However, some regimes indicate wavelike solutions with westward group velocities, enabling Rossby wave wakes to form to the west of islands in this two-layer flow. These regimes only occur when the background state is baroclinically unstable and the PV gradient is reversed in each layer (indicated by the thin grey contours in Figs. 2 and 3). In these cases, the upstream boundary conditions of Section 2 should be applied to the east of the island for that mode.

\section{Some specific examples}

The generation of specific solutions by the adjustment of a quasigeostrophic flow past an island is considered in this section. The amplitudes of the generated modes is determined by the boundary conditions, as described in Section 2. Previous work has illustrated the influence of changing the size of the island (of radius $a$ ) on the quasigesotrophic adjustment (White 1971; Tansley and Marshall 2001), which is governed by the ratio of the lengthscale 
of the modal solution compared with that of the island (the Island parameter). In our two-mode solution, we have two Island parameters:

$$
\mathrm{Is}_{A}=a k_{A} \quad \text { and } \quad \mathrm{Is}_{B}=a k_{B} .
$$

If the Island parameter for a wavelike mode is much less than one, then flows near to the island are close to potential, and the influence of the arrested Rossby wave is negligible. In what follows, we fix the island diameter at $100 \mathrm{~km}$ but vary the latitudes and flow speeds of each layer. For the parameter regimes in this section, the modal lengthscale for both solutions are comparable with that of the island, and Rossby wave solutions are important at the island scale.

We validate our specific solutions using a two-layer, time-dependent quasigeostrophic numerical model. The spectral model solves the time-dependent, coupled equations (Eq. 2) in a doubly periodic domain and enforces the boundary condition at the island using a Greens function method (Cummins and Mysak 1988). Filtering is applied to remove small lengthscales generated by the downscale cascade of enstrophy, and sponges are applied at the boundaries to absorb outgoing wave energy. All simulations presented here have been tested to ensure that the following results are independent of resolution, sponge parameters, and domain size.

\section{a. Eastward sheared flow}

We consider flow past an island of diameter $100 \mathrm{~km}$ at a latitude of $10^{\circ} \mathrm{N}$, in a depth of 3,000 $\mathrm{m}$ and with a layer interface at $200 \mathrm{~m}$. The density difference between the upper and lower layers is $4 \mathrm{kgm}^{-3}$. This parameter regime is inspired by the structure of North Equatorial current close to the island of Palau in the Western Pacific (Schönau and Rudnick 2015) and broadly covers the many small islands in the region. The baroclinic deformation radius is $106 \mathrm{~km}$, corresponding to the regime diagram in Figure 2, and the background speeds are set to $0.1 \mathrm{~ms}^{-1}$ in the upper layer and $0.07 \mathrm{~ms}^{-1}$ in the lower layer. Figure 4 illustrates the modal solutions for these parameters, both of which are wavelike, with the barotropic-like wave having a smaller lengthscale $\left(k_{A}=1.8 \times 10^{-5} \mathrm{~m}^{-1}\right)$ than the baroclinic-like mode $\left(k_{B}=1.3 \times 10^{-5} \mathrm{~m}^{-1}\right)$. The perturbation displacements of the free surface $\left(Z_{0}\right)$ and layer interface $\left(Z_{1}\right)$ are calculated from the streamfunctions in each layer so that

$$
Z_{0}=\frac{f_{0} \phi_{u}}{g}, \quad Z_{1}=\frac{f_{0}\left(\phi_{l}-\phi_{u}\right)}{g^{\prime}} .
$$

The barotropic-like mode dominates the flow structure in the lower layer, whereas the baroclinic-like mode dominates in the upper layer. The resulting layered solutions seem almost uncoupled, qualitatively obeying the single-layer prediction that larger flow speed arrests a longer-wavelength wave in each layer.

The group velocities of the arrested plane waves for the aformentioned parameters are illustrated by a Lighthill diagram (Fig. 5). Wavenumbers associated with the zero frequency 

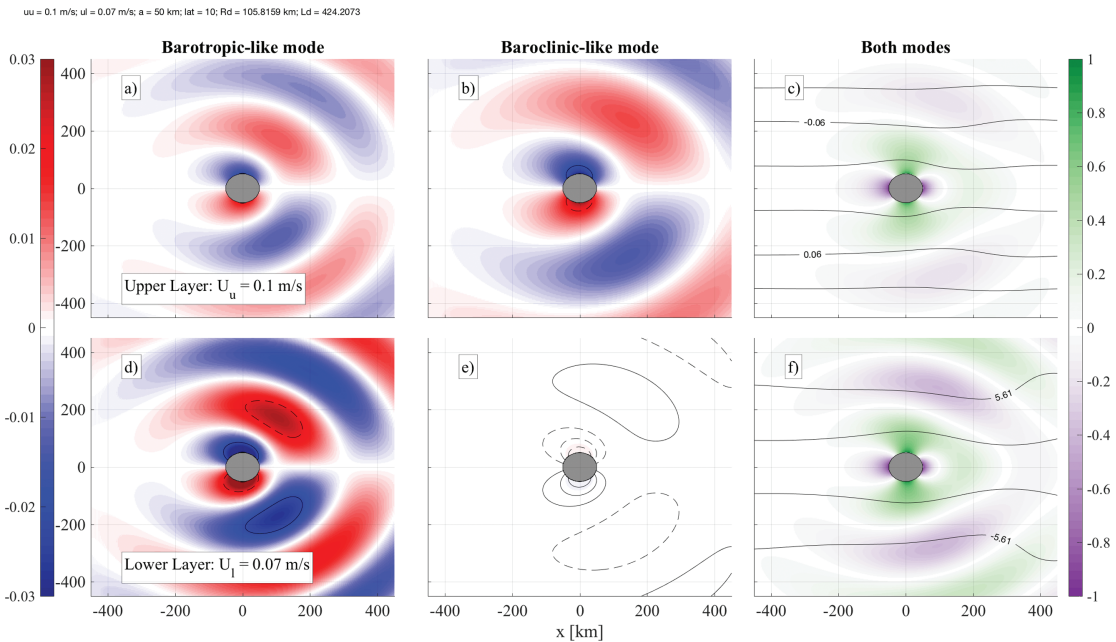

Figure 4. Theoretical prediction for quasigeostrophic adjustment of eastward sheared flow past an island of diameter $100 \mathrm{~km} . R_{D}=106 \mathrm{~km}, u_{u}=0.1 \mathrm{~ms}^{-1}$ and $u_{l}=0.07 \mathrm{~ms}^{-1}$. Colors are normalized vorticity in panels $\mathrm{a}, \mathrm{b}, \mathrm{d}$, and e. Contours are displacement of free surface $(\mathrm{a}, \mathrm{b}$ : intervals $0.005 \mathrm{~m}$ ) and layer interface (d, e; intervals $0.5 \mathrm{~m}$ ): solid lines positive. Panels $\mathrm{c}$ and $\mathrm{f}$ are zonal velocity normalized by background value in each layer (color), and free surface and interface height (contours [m]).
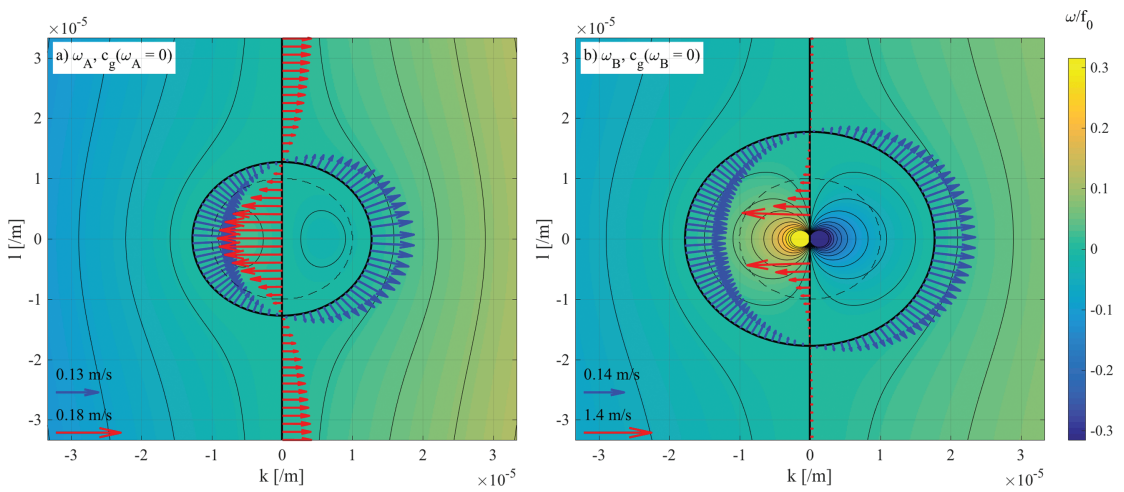

Figure 5. Lighthill diagrams for each mode when the background flow is eastward and sheared. $R_{D}=106 \mathrm{~km}, u_{u}=0.1 \mathrm{~ms}^{-1}$, and $u_{l}=0.07 \mathrm{~ms}^{-1}$. Colors indicate the frequency of waves having wavenumbers $(k, l)$, and arrows indicate the group velocities of the zero frequency (arrested) wave. Group velocity vectors $>0.8 \mathrm{~ms}^{-1}$ near $l=0$ in panel $\mathrm{b}$ are not shown for clarity. The dashed circle indicates the scale of the island in Figure 4.

response are contoured in black, and their associated group velocities are the gradients of the colored (frequency) background in $k, l$ space, indicated with arrows. The Bessel function modes of Section 2 appear as circles of radii $k_{A}$ and $k_{B}$ in wavenumber space for plane waves, and both are associated with eastward group velocities. The $k=0$ mode explicitly 


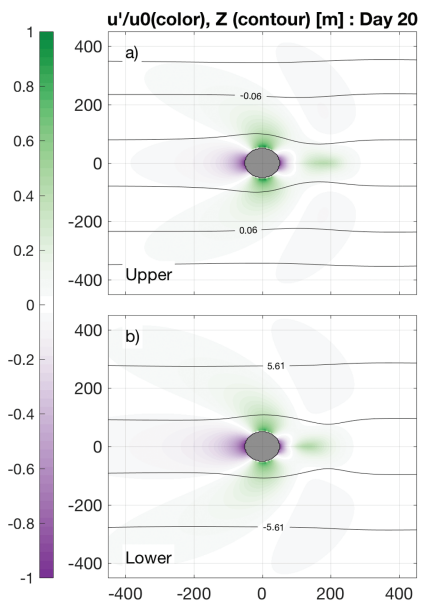

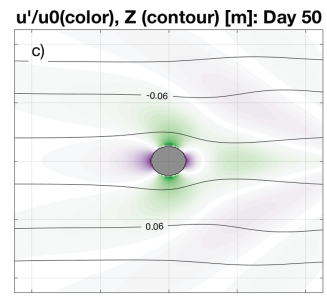

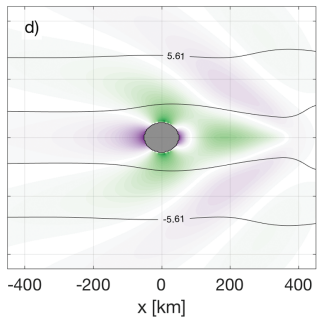

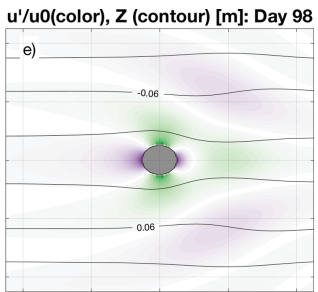

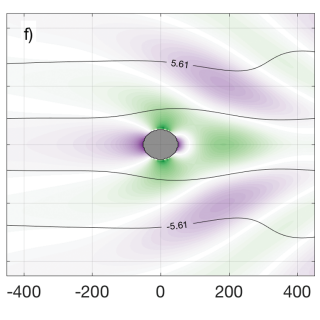

Figure 6. 2-layer QG simulation. $R_{D}=106 \mathrm{~km}, u_{u}=0.1 \mathrm{~ms}^{-1}$, and $u_{l}=0.07 \mathrm{~ms}^{-1}$. Interface heights contoured in $\mathrm{m}$, zonal speeds in color, normalized by background flow speed in each layer.

appears and is associated with fast westward group velocities at large scales and slow eastward group velocities at small scales. In a time-dependent problem, these modes will set the zonal flow upstream and downstream of the island, leading to Lighthill blocking (Rhines 2007). At the scale of the island (indicated with the dashed line), we anticipate blocking modes to extend to the west of the island.

The analytic predictions above are tested in a two-layer quasigeostrophic numerical simulation. The steady state solution with no $k=0$ mode of Section 2 is tested by initializing the flow with the analytic solution, and allowing the simulation to evolve in time. No frictional terms are included in the simulation, other than sponging, required at the periodic boundaries to prevent the generation of a basin standing mode. Consistent with the initial condition being a steady solution, the numerical simulation is steady and evolves only very slowly over a 100-day simulation due to the influence of boundary effects and (small) numerical diffusion in the domain interior. The careful initialization of the simulation with the analytic solution means that there is no adjustment to the upstream flow by blocking modes, and it remains zonally uniform.

In a second simulation we initialize the simulation with a quiescent flow, but ramp it up over the first 3 days of the simulation to a steady geostrophic background flow, watching the adjustment of the flow to the island boundary conditions (Fig. 6). A Laplacian diffusivity of $2.3 \mathrm{~m}^{2} \mathrm{~s}^{-1}$ is employed in this simulation to maintain stability, as the adjustment process generates small scales close to the island boundary. We anticipate that this time-dependent problem will include transient wave responses, specifically where $\omega \neq 0$, nevertheless the overall response downstream of the island is well predicted by the two steady wavelike modes within a few days of starting the simulation, with the wave scales predicted by theory 
in Section 2 emerging in each layer and extending eastward with time. Group speeds are comparable with those predicted in Figure 5, with values of around $0.15 \mathrm{~ms}^{-1}$. Directly upstream of the island, zonal flow is arrested in a blocked region, which extends westward, weakening zonal flow in a meridional band centered on the island. The spatial structure of zonal velocity along the centerline of the island is exponential, with a length scale of approximately $50 \mathrm{~km}$ such that the flow is within $2 \%$ the background $(0.1 \mathrm{~m} / \mathrm{s}) 400 \mathrm{~km}$ upstream of the island. This part of the upstream solution is well predicted by the solutions of Section 2, indicating that this stagnant region is associated with the wavelike modes. Upstream to the north and south, flows are accelerated nearly $20 \%$ relative to the background and have velocity profiles that decay nearly linearly towards the background flow at the edge of the domain. By 100 days, they are still somewhat unsteady, growing at around $1 \%$ per day. These structures are not predicted by the wavelike modes of Section 2, and we associate them with $k=0$ Lighthill modes. In our model we expect them to eventually be diffusively balanced. The meridional structure of the blocking modes is matched to that of the wavelike modes at the island, with alternating bands of zonal velocity extending meridionally to the boundaries of the simulation. Blocking modes are also present to the east of the island by day 50 of the simulation, where a narrow, jet-like feature is apparent in both layers around $y=0, x=500 \mathrm{~km}$ (Fig. 6c), corresponding to the predicted eastward $k=0$ mode at small meridional scales. Over the course of the simulation the feature continues to extend eastward, leading to a weak jet along the centerline of the island compared with upstream.

\section{b. Westward sheared flow}

Stratification is the same as in the eastward sheared flow, but we increase the latitude of the island to $25^{\circ} \mathrm{N}$ so $R_{D}=43 \mathrm{~km}$, corresponding to the regime diagram in Figure 3 . We prescribe westward flow in each layer, with $u_{u}=-0.1 \mathrm{~ms}^{-1}$ and $u_{l}=-0.07 \mathrm{~ms}^{-1}$. In this case both modal solutions are evanescent, but the layers are clearly coupled with both modes having signatures in each layer (Fig. 7). Compared with the eastward sheared flow, layer displacements close to the island are much larger, reaching up to $4 \mathrm{~m}$ in the lower layer compared with $1.5 \mathrm{~m}$ in the eastward flow.

Group velocities for arrested modes are indicated in Figure 8 and show that Lighthill blocking at all scales is expected to develop to the west of the island. The fastest signals have scales larger than the island itself, and the blocking would be expected to develop more meridional structure in time, as the shorter scales propagate downstream.

As in the eastward sheared case we test the analytic solution in the time-dependent model. By initializing the simulation with the analytic solution, the model is steady over a 100-day simulation, confirming the validity of the analytic solution. The role of timedependent waves are apparent when the simulation is initialized with zero background flow, then ramped up to steady values over the first three days of the simulation. In this case the transient modes are more prominent than in the eastward sheared case, radiating 

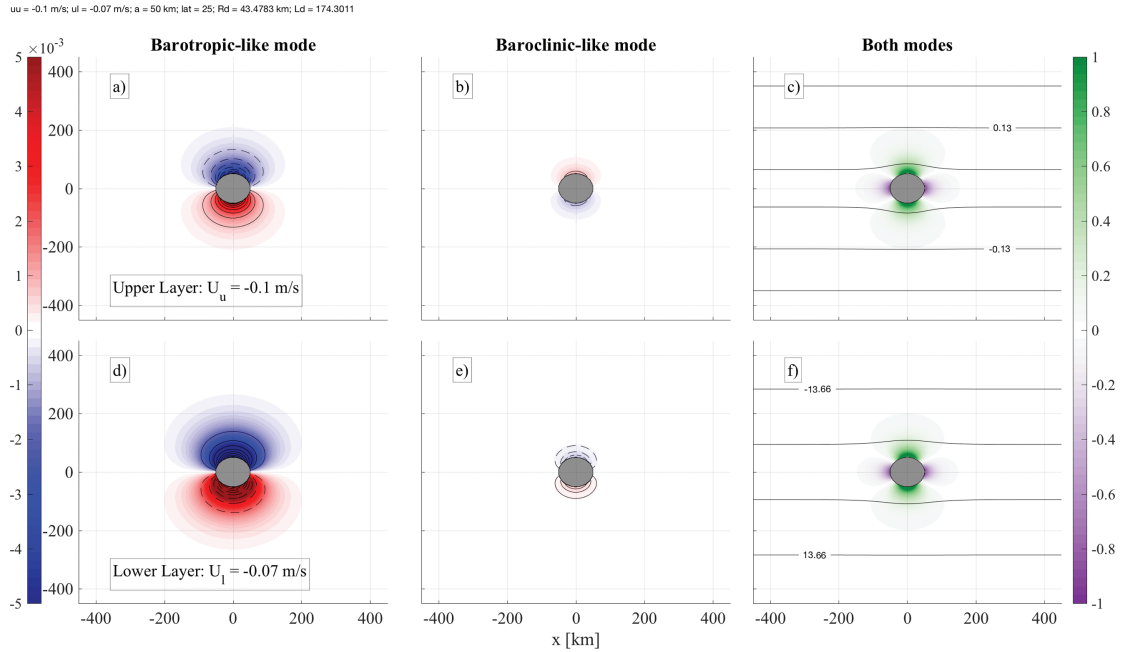

Figure 7. Theoretical prediction for quasigeostrophic adjustment of westward sheared flow past an island of diameter $100 \mathrm{~km} . R_{D}=43 \mathrm{~s} \mathrm{~km}, u_{u}=-0.1 \mathrm{~ms}^{-1}$, and $u_{l}=-0.07 \mathrm{~ms}^{-1}$. Colors are normalized vorticity in panels $\mathrm{a}, \mathrm{b}, \mathrm{d}$, and e. Contours are displacement of free surface $(\mathrm{a}, \mathrm{b}$ : intervals $0.005 \mathrm{~m}$ ) and layer interface (d, e: intervals $0.5 \mathrm{~m}$ ): solid lines positive. Panels $\mathrm{c}$ and $\mathrm{f}$ are zonal velocity normalized by background value in each layer (color), and free surface and interface height (contours $[\mathrm{m}]$ ).
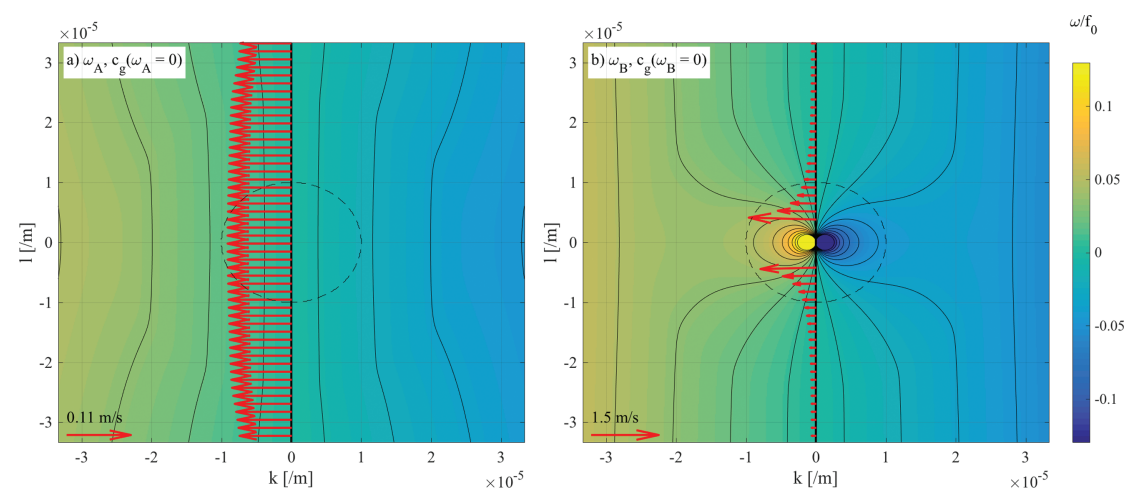

Figure 8. Lighthill diagrams for each mode when the background flow is westward and sheared across the two layers. $\mathrm{Rd}=43 \mathrm{~km}, u_{u}=-0.1 \mathrm{~ms}^{-1}$, and $u_{l}=-0.07 \mathrm{~ms}^{-1}$. Colors indicate the frequency of waves having wavenumbers $(k, l)$, and arrows indicate the group velocities of the zero frequency (arrested) wave. Group velocity vectors $>0.8 \mathrm{~ms}^{-1}$ near $l=0$ in panel $\mathrm{b}$ are not shown for clarity. The dashed circle indicates the scale of the island in Figure 7.

meridionally as they propagate westward out of the domain (Fig. 9). The remaining flow at 100 days near the island is qualitatively similar to the analytic solution, with an evanescent acceleration of the westward flow at the meridional tips of the island. 

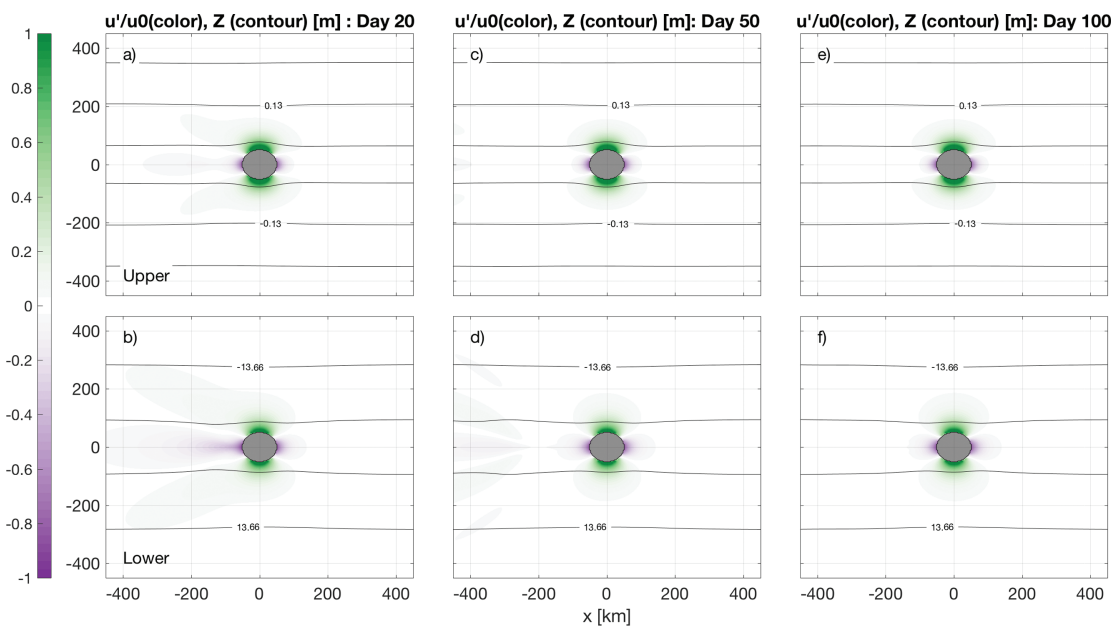

Figure 9. 2-layer QG simulation. $R_{D}=43 \mathrm{~km}, u_{u}=-0.1 \mathrm{~ms}^{-1}$, and $u_{l}=-0.07 \mathrm{~ms}^{-1}$. Interface heights contoured in $\mathrm{m}$, zonal speeds in color, normalized by background flow speed in each layer.

\section{c. Rossby wave formation to the west of the island}

An examination of plane wave zonal group velocities in Figures 2 and 3 suggest that, under some parameter regimes, the solutions for flow past the island may also give rise to wavelike modes with westward energy propagation. We test this prediction in the time-dependent quasigeostrophic numerical model, choosing parameters from Figure 3 of $u_{u}=-0.1 \mathrm{~ms}^{-1}$ and $u_{l}=0.2 \mathrm{~ms}^{-1}$, which predict the barotropic-like mode to be wavelike with small scales and an analogous plane wave having westward group velocity and the barocliniclike mode to be wavelike with large scales and an analogous plane wave having eastward group velocity. The time-dependent evolution of the flow from a zonal geostrophic initial state is shown in Figure 10. Gradients in the background potential vorticity are reversed in each layer, and the background state is baroclinically unstable. The time evolution of the instability is apparent as potential vorticity anomalies grow in amplitude over the 100 days; however, the Rossby wave wake predicted from the steady solution becomes apparent much sooner, on a timescale associated with the group velocities and wavelengths of the waves. Typical establishment times for a Rossby wave wake with a group velocity of $1 \mathrm{~m} / \mathrm{s}$ and lengthscale of $300 \mathrm{~km}$ are around 4 days, which is faster than estimated Eady growth rates in many parts of the ocean (Tulloch et al. 2011). Rossby wave wakes are apparent in both layers with the barotropic-like mode dominant in the upper layer and developing a Rossby wave to the west of the island. The baroclinic-like mode has a larger lengthscale and signatures in both layers and evolves on the timescale of the instability. By day 98 the developing baroclinic instability dominates to the east of the island, where the Rossby wave wake has grown to large amplitude and is somewhat unsteady. 


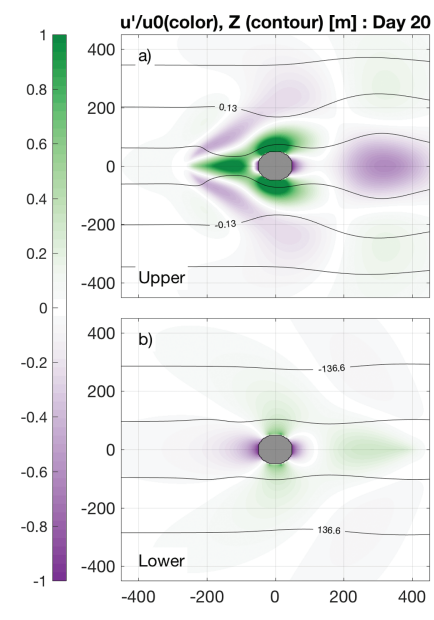

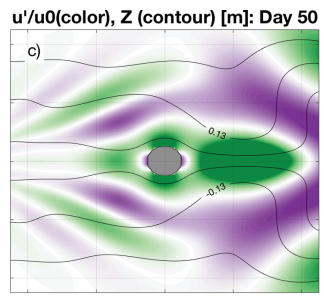

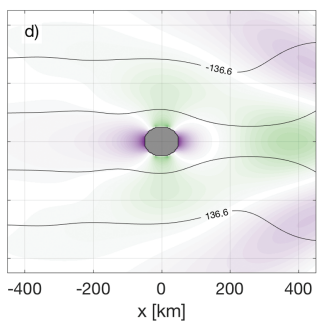

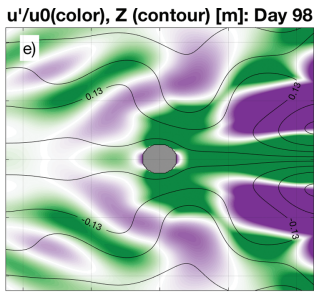

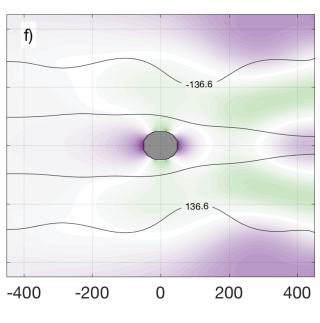

Figure 10. 2-layer QG simulation. $R_{D}=43 \mathrm{~km}, u_{u}=-0.1 \mathrm{~ms}^{-1}$, and $u_{l}=0.2 \mathrm{~ms}^{-1}$. PV in lower layer has been multiplied by 5 .

\section{The formation of wake eddies}

The influence of friction at the boundary of the island provides a no-slip boundary condition, setting the gradient of the streamfunction at the boundary, $\psi_{r}=0$. The frictional boundary layer that arises with this condition was considered in a homogeneous fluid by Merkine (1980), who used an inviscid outer solution matched to a viscous inner solution near to the island to examine the role of a Rossby wave wake on the boundary layer. In westward flows the boundary layer solution close to the island is uninfluenced by rotation, and leads to flow separation close to the meridional tips of the island as in the classic problem of unstratified flow past a bluff body (Gerrard 1978; Van Dyke 1982). However, in eastward flows the boundary layer solution close to the island is modified by the arrested Rossby wave, whose distribution of pressure near the island shifts the adverse pressure gradient in the boundary layer downstream, delaying separation of the boundary layer from the island. In turn, this inhibits the formation of island wake eddies, which acquire their vorticity from the frictional boundary layer. The theoretical prediction of Merkine (1980) was validated in a series of laboratory experiments by Boyer and Davies (1982), who clearly illustrated the arrest of Rossby waves and inhibition of wake eddy generation for eastward flows.

Though these results were obtained through consideration of a homogeneous fluid, it is clear that the same mechanism for wake inhibition can arise in the two-layer solution, as the formation of an arrested Rossby wave mode alters the pressure gradient near to the island boundary. Terms like $\sin 2 \theta$ in Eq. (19) are asymmetric around the island, leading to an acceleration of the flow downstream of the island that is associated with lower pressure. However, the vertical structure of the flow in the two-layer problem enables different wake properties in each layer, determined by the near-island pressure gradient in the layer. 
In a series of numerical experiments, Dong and McWilliams (2007) consider continuously stratified flow past a cylindrical island. They do not consider the effects of $\beta$ and, as such, neglect the influence of an arrested Rossby wave on the boundary layer. Nevertheless, we expect their results to apply when the quasigeostrophic modes are evanescent. They show that at sufficiently high Reynolds numbers $(\operatorname{Re}=U L / \nu \gtrsim 100$, where $U$ is the velocity scale, $L$ the lengthscale, and $\nu_{e}$ the explicit viscosity used in their simulations) the separated boundary layers roll up into a series of submesoscale island wake vorticies, initially having qualitative similarities to those in an unstratified flow, but subject to a range of rotational and stratified instabilities.

In this section, the Hallberg Isopycnal Model (HIM; Hallberg 1997) is used to examine the influence of both nonquasigesotrophic effects and frictional boundary conditions in our solutions. The model is configured with two layers in a domain that is periodic in the zonal and walled in the meridional direction. The underlying geometry is spherical-domains are zonal channels on the sphere. Initial conditions are uniform, $f$-plane geostrophically balanced flow in each layer, and the flow is restored to these initial conditions in sponge regions at all boundaries so that outward radiating energy is damped, and the simulation mimics an infinite domain. Resolution is approximately $2 \mathrm{~km}$, and a Laplacian eddy diffusivity of $12 \mathrm{~m}^{2} \mathrm{~s}^{-1}$ is employed to maintain stability, giving an overall $\mathrm{Re} \approx 1,000$. No-slip boundary conditions are applied at walls of the cylindrical island, but simulations with nostress (i.e., $\psi_{r r}=0$ ) at the island boundary yield very similar results and are not presented here. We discuss results from simulations with flow parameters for the eastward sheared and westward sheared flows examined in Section 4. Both simulations are run for 200 days.

\section{a. Eastward sheared flow}

A Rossby wave wake forms to the east of the island, with scales and amplitudes that are consistent with the results from the quasigeostrophic simulation of the previous section (Fig. 11). The key difference between the simulations arises from the no-slip boundary conditions applied in HIM, which form a source of relative vorticity in the simulation that is about 10 times greater than that generated by the formation of the Rossby wave. Recirculating eddies form to the east of the island close to its central latitude (i.e., away from the meridional extremities) on a timescale of a few days after the simulation initializes. However, they remain attached to the island and the vorticity associated with them forms extended streamers along the central meridian downstream. This vorticity is of the opposite sign to that established by the downstream blocking, causing the downstream blocking jet to be meridionally separated by a relatively quiescent region, with split jets to the north and south. The jets meander downstream of the island (around $x=400 \mathrm{~km}$ ), possibly as a result of barotropic instability (Fig. 11e.).

\section{b. Westward sheared flow}

As predicted by the quasigeostrophic model, the larger scale flow past the island in this case is evanescent. However, the response is dominated by the generation of wake eddies 

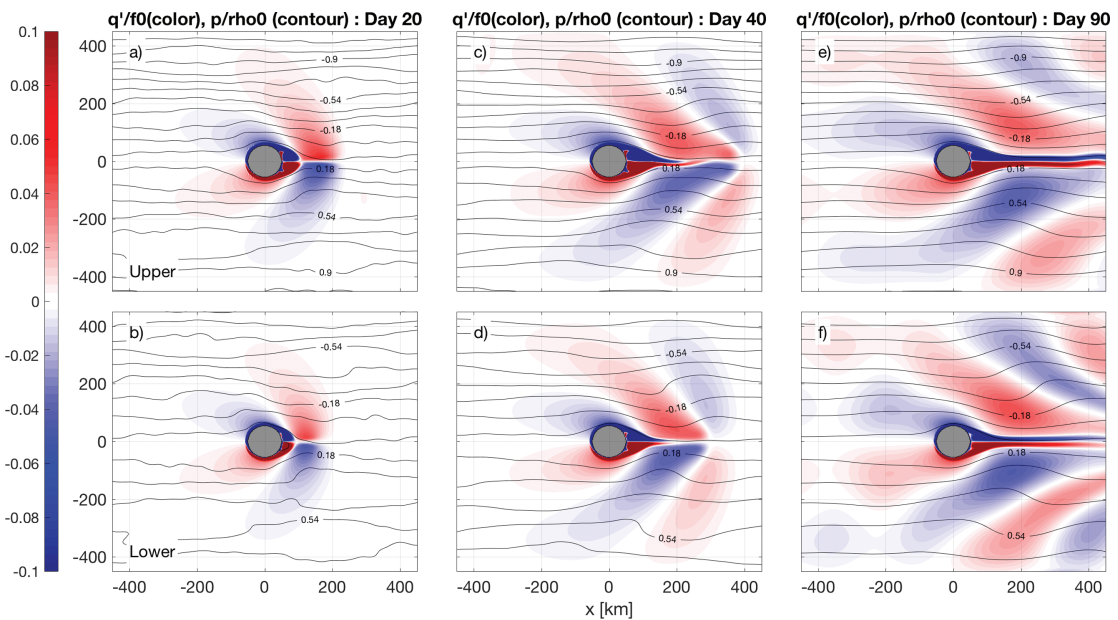

Figure 11. 2-layer full physics simulation. $\mathrm{Rd}=106 \mathrm{~km}, u_{u}=0.1 \mathrm{~ms}^{-1}$, and $u_{l}=0.07 \mathrm{~ms}^{-1}$. Five-day averaged values of perturbation Ertel PV anomaly, $q^{\prime}=\xi / h_{i}$, in color. Dynamic pressure contoured.
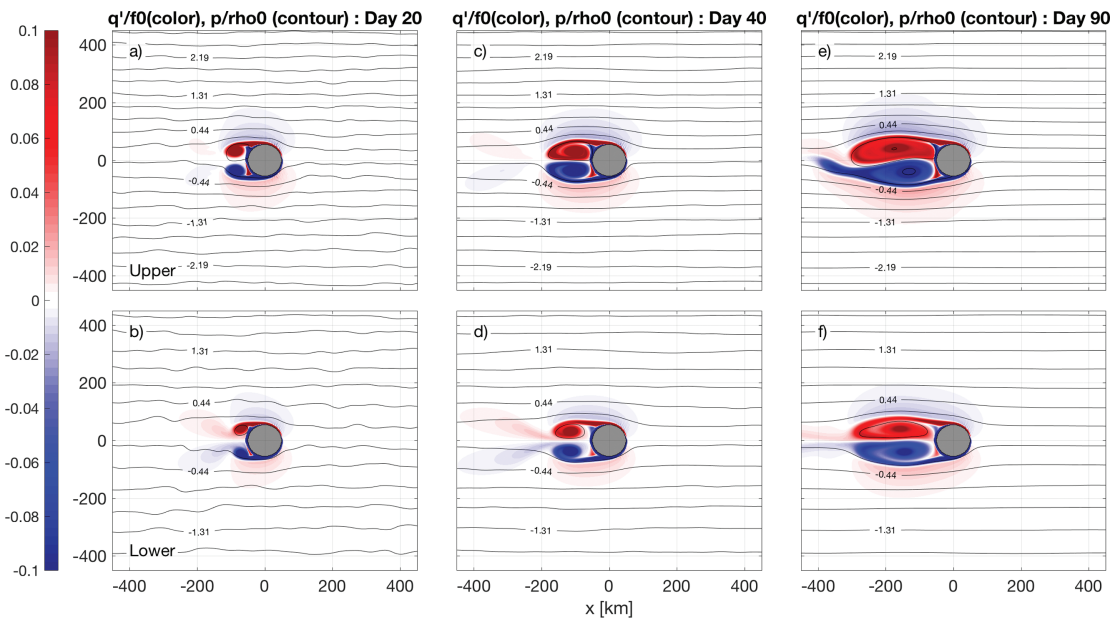

Figure 12. 2-layer full physics simulation. $\mathrm{Rd}=43 \mathrm{~km}, u_{u}=-0.1 \mathrm{~ms}^{-1}$, and $u_{l}=-0.07 \mathrm{~ms}^{-1}$. Five day averaged values of perturbation Ertel PV anomaly, $q^{\prime}=\xi / h_{i}$, in color. Dynamic pressure contoured.

at the northern and southern tips of the island (Fig. 12). Near to the island the vorticity associated with the boundary layers is of the opposite sign to that associated with the quasigeostrophic response, and, as in the eastward sheared case, it dominates the signal. The wake eddies develop from the northern and southern tips of the island within the first 
20 days of the simulation and grow downstream in time, eventually separating and forming an unsteady von Kármán vortex street after about 180 days (not shown).

Consistent with our expectations from earlier work, the role of $\beta$ on wake eddies is dramatic for these small islands in zonal background flows. Though the amplitude of the quasigeostrophic response is relatively small and difficult to directly measure in the ocean, the influence of the quasigeostrophic response on the island wake is significant, qualitatively changing the structure of the wake and leading to a tendency for wakes in the presence of an arrested Rossby wave to remain fully attached (Boyer and Davies 1982).

\section{c. Island wakes and drag}

The zonal pressure drag on a cylinder is the integral of the pressure force on the cylinder in the zonal direction:

$$
F_{D}=\oint p(\theta) \cos \theta d \theta
$$

In the solutions of Section 2, wave drag occurs at order $\mathrm{Ro}^{2}$ and is estimated from the quasigeostrophic solution as

$$
F_{D}=-\rho_{0} \frac{1}{2} \oint \psi_{r}^{2} \cos \theta d \theta
$$

In these inviscid solutions, drag is only anticipated when a Rossby wave is arrested in the lee of the island: in this case the pressure distribution is zonally asymmetric as flow accelerates on the downstream side, leading to a pressure drop over the island. Drag due to the blocking modes is sensitive to the initial conditions of the flow, and is not considered here; however, in general they may have an influence. The sign of the Rossby wave drag depends on the location of the wave with respect to the island, i.e., the direction of the group velocity. Drags from the modal solutions with parameters in Figures 2 and 3 are presented in Figure 13, where the eastward or westward formation of the Rossby wave was determined using the group velocities of plane waves. Depth mean drag is largely influenced by flow speed and structure in the lower layer at these parameters (lower layer depth is much larger than the upper layer), and exhibits small jumps between regions where one or two Rossby waves are arrested by the island. Regions of positive drag appear when the group velocities of both modes are expected to be westward, corresponding to arrested lee waves forming to the west of the island.

The addition of friction at the island boundary, however, leads to a drag even when both arrested modes are evanescent as boundary layer separation and the formation of island wake eddies dominates drag processes at the island.

Pressure drag was calculated in each of the two simulations in HIM by evaluating Eq. (27) in each of the layers averaged over 20 days when the simulation reached steady state (in the westward flow this corresponds to about one eddy shedding period). Depth mean drags were larger in the westward flow, exerting $30 \mathrm{~N} \mathrm{~m}^{-2}$ on the island, compared with the the 

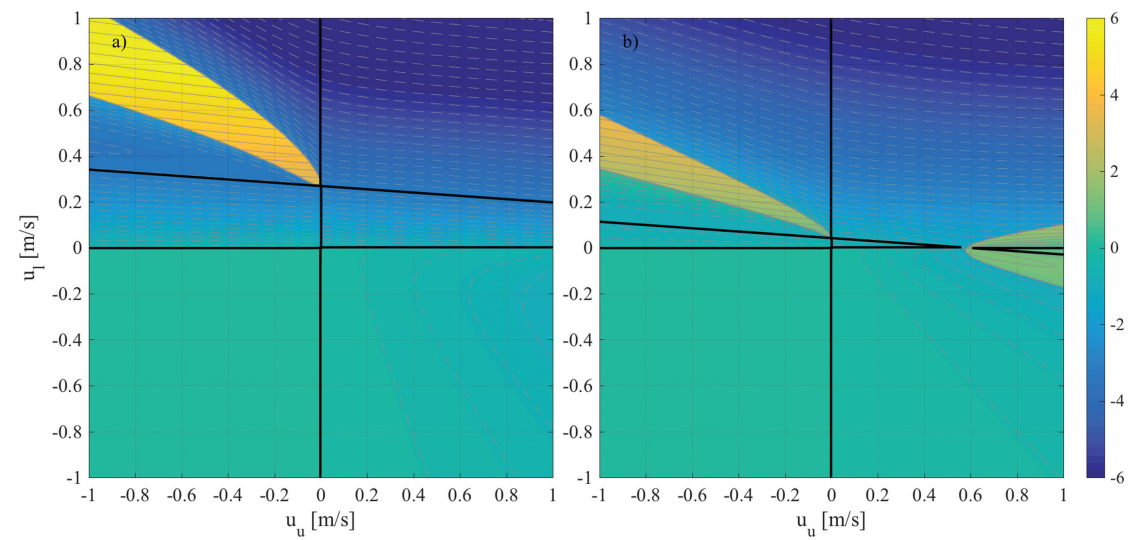

Figure 13. Depth mean form drag (color and grey contours, $\mathrm{N} / \mathrm{m}^{2}$ ) from inviscid quasigeostrophic solutions. a) $R_{D}=106 \mathrm{~km}$, b) $R_{D}=43 \mathrm{~km}$. Black contours indicate where modal solutions transition from wavelike to evanescent (refer Figs. 2 and 3 for corresponding lengthscales of arrested waves).

eastward flow, which exerted $-14 \mathrm{~N} \mathrm{~m}^{-2}$. The contribution to the drag from Rossby waves for the eastward configuration was estimated analytically to be $-2.8 \mathrm{~N} \mathrm{~m}^{-2}$. Despite the presence of wave drag in the eastward flow configuration, the overall drag was reduced compared with the westward flow as a result of inhibited flow separation and island wake formation.

\section{Summary}

Zonal flow past a cylinder on a $\beta$-plane has relevance to the interaction of large-scale geostrophic flows with small islands. Our study and parameter space is motivated by the interaction of the North Equatorial Current with the island of Palau in the equatorial Pacific; however, our results are applicable over a wide range of parameters where friction and forcing drive no net circulation. The interface between the two layers crudely mimics a strong pycnocline and provides a first step in understanding the role of stratification on island flow interactions. Arrested Rossby waves are relevant when the Island number is greater than one, indicating that the scale of the arrested wave is comparable with or smaller than the size of the island. For typical oceanic parameters, this can be true for islands of only $100 \mathrm{~km}$ diameter. These quasigeostrophic solutions set the structure of the large-scale flow near to the island, influencing nonquasigeostrophic processes such as island wake shedding.

Analytic solutions for the two layer system were found in a quasigeostrophic framework with the upstream flow specified to be uniform and zonal, and no flow through the island boundary. The solutions are formed from the sum of arrested eigenmodes in the 2 layer quasigeostrophic system, where the eigenmodes can be either wavelike or evanescent, depending on background flow conditions (layer speeds, stratification, and latitude). 
In general, the eigenmodes cannot be simply described as barotropic or baroclinic but have a mixed nature. The baroclinic-like solution exhibits a supercritical transition from wavelike or evanescent behavior when the phase speed of the mode is insufficient to match the oncoming flow. The transition depends on the the Rossby deformation radius and $L_{\beta}$ : for eastward flow in both layers, the baroclinic-like mode is wavelike when $R_{D}>L_{\beta}$ and evanescent when $R_{D}<L_{\beta}$. In this two-layer flow, arrested Rossby waves can be associated with group velocities that are both eastward and westward, enabling the generation of a Rossby wave wake to the west of the island.

Upstream influence by the island is communicated by blocking modes and, in general, must be considered during the establishment of steady flows. In the ocean these modes are approximated by unsteady modes with $\omega \approx 0$ and exhibit characteristics close to the purely steady modes with analogous $k \approx 0$ modes and fast group velocities (Baines and Hughes 2016). If the background flow becomes steady, the upstream/downstream adjustments manifest in the steady solution as $k=0$, or Lighthill blocking modes. Despite prescribing the upstream state in our analytic solution, we find that it predicts the downstream structures in time-dependent simulations that allow blocking to alter the upstream flow.

Our island wake solutions exhibit many similarities to the seamount solution of McCartney (1975), including the transition from wavelike to evanescent behavior under specific parameter regimes. However, the form of the solutions are quite different near to topography as the flow is accelerated over the seamount, rather than deflected around the island. This distinct behavior is described by cosine modes appearing in the seamount solutions rather than the sine modes of the island problem; the former may project more strongly onto the upstream and downstream blocking Lighthill modes.

Full physics numerical simulations in two layers indicate that the presence of an arrested Rossby wave can influence submesoscale island wake formation on timescales of $10 \mathrm{~s}$ of days. The pressure distribution associated with an arrested Rossby wave inhibits flow separation in the viscous boundary layer at the island and prohibits the generation of island wake eddies in our simulations. In general the formation of island wake eddies is Reynolds number dependent, with separation occurring sooner and wakes becoming more chaotic at high Reynolds number. We expect a similar behavior for island wake formation in an arrested Rossby wave, but we anticipate that island wakes in this case will be smaller and confined closer to the central meridian of the island than when island wakes form in a quasigeostrophic evanescent flow.

Rossby wave drag plays a small role in the overall drag on the flow by the island, accounting for less than $20 \%$ of the pressure drag at the island in the specific eastward sheared flow configuration. Pressure drag was dominated by the separation of the viscous boundary layer downstream of the island and was largest in the westward sheared flow configuration where island wake eddies formed in the lee. The inhibition of island wake formation in the presence of an arrested Rossby wave led to a reduction of the overall pressure drag in the simulations that we compared. This occurred despite the direct contribution of the Rossby wave to the drag. 
Acknowledgments. RCM thanks Larry Pratt for many useful and encouraging conversations over the course of this work. This work was supported by the Office of Naval Research Program 322, Physical Oceanography, Grant Number N000141512622.

\section{Appendix A: Two wavelike modes}

The general solution for two wavelike modes is

$$
\begin{aligned}
{\left[\begin{array}{c}
\phi_{u} \\
\phi_{l}
\end{array}\right]=} & {\left[\begin{array}{c}
1 \\
\Delta_{A}
\end{array}\right]\left(\left[a_{A n} \cos n \theta+b_{A n} \sin n \theta\right] J_{n}\left(k_{A} r\right)+\left[c_{A n} \cos n \theta+d_{A n} \sin n \theta\right] Y_{n}\left(k_{A} r\right)\right) } \\
& +\left[\begin{array}{c}
1 \\
\Delta_{B}
\end{array}\right]\left(\left[a_{B n} \cos n \theta+b_{B n} \sin n \theta\right] J_{n}\left(k_{B} r\right)+\left[c_{B n} \cos n \theta+d_{B n} \sin n \theta\right] Y_{n}\left(k_{B} r\right)\right) .
\end{aligned}
$$

The lowest order term is $n=1$, where

$$
\phi_{1}=\left[\begin{array}{c}
1 \\
\Delta_{A}
\end{array}\right]\left[b_{A 1} J_{1}\left(k_{A} r\right)+d_{A 1} Y_{1}\left(k_{A} r\right)\right] \sin \theta+\left[\begin{array}{c}
1 \\
\Delta_{B}
\end{array}\right]\left[b_{B 1} J_{1}\left(k_{B} r\right)+d_{B 1} Y_{1}\left(k_{B} r\right)\right] \sin \theta .
$$

The boundary condition $\psi_{I}=-u a \sin \theta+\phi=0$ imposed at $r=a$ gives

$$
\begin{aligned}
& b_{B 1} J_{1}\left(k_{B} a\right)=\frac{\left(u_{l}-\Delta_{A} u_{u}\right) a+d_{B 1} Y_{1}\left(k_{B} a\right)\left(\Delta_{A}-\Delta_{B}\right)}{\Delta_{B}-\Delta_{A}} \\
& b_{A 1} J_{1}\left(k_{A} a\right)=\frac{\left(u_{l}-\Delta_{B} u_{u}\right) a+d_{A 1} Y_{1}\left(k_{A} a\right)\left(\Delta_{B}-\Delta_{A}\right)}{\Delta_{A}-\Delta_{B}} .
\end{aligned}
$$

$n \geq 2$

The boundary condition, $\psi_{I}=-u a \sin \theta+\phi=0$ at $r=a$ gives

$$
\begin{gathered}
b_{A n} J_{n}\left(k_{A} a\right)+d_{A n} Y_{n}\left(k_{A} a\right)+b_{B n} J_{n}\left(k_{B} a\right)+d_{B n} Y_{n}\left(k_{B} a\right)=0 \\
\Delta_{A}\left[b_{A n} J_{n}\left(k_{A} a\right)+d_{A n} Y_{n}\left(k_{A} a\right)\right]+\Delta_{B}\left[b_{B n} J_{n}\left(k_{B} a\right)+d_{B n} Y_{n}\left(k_{B} a\right)\right]=0,
\end{gathered}
$$

which combine to give

$$
\begin{aligned}
& b_{B n} J_{n}\left(k_{B} a\right)=-d_{B n} Y_{n}\left(k_{B} a\right) \\
& b_{A n} J_{n}\left(k_{A} a\right)=-d_{A n} Y_{n}\left(k_{A} a\right) .
\end{aligned}
$$

The modal solutions should not radiate energy in the direction upstream of the island, i.e., in the direction opposite to the group velocity of the mode. For modes having an eastward group velocity, the condition requires that $r^{1 / 2} \phi(r \rightarrow \infty)=0$ for $\pi / 2<\theta<3 \pi / 2$. We approximate this condition, following the method of White (1971), by specifying that $r^{1 / 2} \partial \phi / \partial r \mid(r \rightarrow \infty)=0$ for $\theta=\pi$. Using the asymptotic forms for the Bessel functions as $r \rightarrow \infty$, the conditions become 


$$
\begin{aligned}
\left.\frac{\partial \phi}{\partial \theta}\right|_{\theta=\pi}= & \gamma_{A}\left[\begin{array}{c}
1 \\
\Delta_{A}
\end{array}\right]\left[b_{A n} n(-1)^{n} \cos \left(r k_{A}+\frac{1}{2} n \pi-\frac{1}{4} n \pi\right)\right. \\
& \left.+d_{A n} n(-1)^{n} \sin \left(r k_{A}+\frac{1}{2} n \pi-\frac{1}{4} n \pi\right)\right]+\gamma_{B}\left[\begin{array}{c}
1 \\
\Delta_{B}
\end{array}\right] \\
& \times\left[b_{B n} n(-1)^{n} \cos \left(r k_{B}+\frac{1}{2} n \pi-\frac{1}{4} n \pi\right)+d_{B n} n(-1)^{n} \sin \left(r k_{B}+\frac{1}{2} n \pi-\frac{1}{4} n \pi\right)\right] \\
= & 0 .
\end{aligned}
$$

where

$$
\gamma_{A}=\sqrt{\frac{2}{\pi r k_{A}}} \quad ; \quad \gamma_{B}=\sqrt{\frac{2}{\pi r k_{B}}} .
$$

Coefficients are found by setting $b_{A n}=d_{A n}=b_{B n}=d_{B n}=0$ for $n \geq 3$. Including higher order terms must be solved approximately as the system becomes overdetermined, however, these terms do not qualitatively affect the solutions. The coefficients become

$$
\begin{array}{ccc}
b_{B 1}=-2 d_{B 2} & ; & b_{A 1}=-2 d_{A 2} \\
d_{B 1}=2 b_{B 2} & ; & d_{A 1}=2 b_{A 2} .
\end{array}
$$

Note that for upstream influence to the east of the island (i.e., at $\theta=0$ ), the signs of the relations in the above expressions are reversed. The constants for upstream to the west of the island are

$$
\begin{aligned}
b_{B 1} & =\frac{\left(u_{l}-\Delta_{A} u_{u}\right) J_{2}\left(k_{B} a\right) a}{\Upsilon_{B}\left(\Delta_{B}-\Delta_{A}\right)} \quad, & b_{B 2} & =\frac{1}{2} \frac{\left(u_{l}-\Delta_{A} u_{u}\right) Y_{2}\left(k_{B} a\right) a}{\Upsilon_{B}\left(\Delta_{B}-\Delta_{A}\right)} \\
d_{B 1} & =\frac{\left(u_{l}-\Delta_{A} u_{u}\right) Y_{2}\left(k_{B} a\right) a}{\Upsilon_{B}\left(\Delta_{B}-\Delta_{A}\right)} \quad, & d_{B 2} & =-\frac{1}{2} \frac{\left(u_{l}-\Delta_{A} u_{u}\right) J_{2}\left(k_{B} a\right) a}{\Upsilon_{B}\left(\Delta_{B}-\Delta_{A}\right)}
\end{aligned}
$$

where $\Upsilon_{B}=J_{1}\left(k_{B} a\right) J_{2}\left(k_{B} a\right)+Y_{1}\left(k_{B} a\right) Y_{2}\left(k_{B} a\right)$, and

$$
\begin{aligned}
b_{A 1} & =\frac{\left(u_{l}-\Delta_{B} u_{u}\right) J_{2}\left(k_{A} a\right) a}{\Upsilon_{A}\left(\Delta_{A}-\Delta_{B}\right)} \quad, & b_{A 2} & =\frac{1}{2} \frac{\left(u_{l}-\Delta_{B} u_{u}\right) Y_{2}\left(k_{A} a\right) a}{\Upsilon_{A}\left(\Delta_{A}-\Delta_{B}\right)} \\
d_{A 1}=\frac{\left(u_{l}-\Delta_{B} u_{u}\right) Y_{2}\left(k_{A} a\right) a}{\Upsilon_{A}\left(\Delta_{A}-\Delta_{B}\right)} & , & d_{A 2} & =-\frac{1}{2} \frac{\left(u_{l}-\Delta_{B} u_{u}\right) J_{2}\left(k_{A} a\right) a}{\Upsilon_{A}\left(\Delta_{A}-\Delta_{B}\right)}
\end{aligned}
$$

with $\Upsilon_{A}=J_{1}\left(k_{A} a\right) J_{2}\left(k_{A} a\right)+Y_{1}\left(k_{A} a\right) Y_{2}\left(k_{A} a\right)$. The full solution is

$$
\begin{aligned}
{\left[\begin{array}{c}
\psi_{u} \\
\psi_{l}
\end{array}\right]=} & {\left[\begin{array}{c}
1 \\
\Delta_{A}
\end{array}\right]\left(\left[b_{A 1} J_{1}\left(k_{A} r\right)+d_{A 1} Y_{1}\left(k_{A} r\right)\right] \sin \theta+\left[b_{A 2} J_{2}\left(k_{A} r\right)+d_{A 2} Y_{2}\left(k_{A} r\right)\right] \sin 2 \theta\right) } \\
& +\left[\begin{array}{c}
1 \\
\Delta_{B}
\end{array}\right]\left(\left[b_{B 1} J_{1}\left(k_{B} r\right)+d_{B 1} Y_{1}\left(k_{B} r\right)\right] \sin \theta+\left[b_{B 2} J_{2}\left(k_{B} r\right)+d_{B 2} Y_{2}\left(k_{B} r\right)\right] \sin 2 \theta\right) \\
& -\left[\begin{array}{c}
u_{u} \\
u_{l}
\end{array}\right] r \sin \theta
\end{aligned}
$$




\section{Appendix B: Mixed solutions}

These occur when $k_{A}^{2}>0, k_{B}^{2}<0$. Writing the wavelike eigenvalue $k_{W}$, and the evanescent $k_{E}$, then the general solution is

$$
\begin{aligned}
{\left[\begin{array}{c}
\phi_{u} \\
\phi_{l}
\end{array}\right]=} & {\left[\begin{array}{c}
1 \\
\Delta_{W}
\end{array}\right]\left(\left[b_{W n} \sin n \theta\right] J_{n}\left(k_{W} r\right)+\left[d_{W n} \sin n \theta\right] Y_{n}\left(k_{W} r\right)\right) } \\
& +\left[\begin{array}{c}
1 \\
\Delta_{E}
\end{array}\right]\left[d_{E n} \sin n \theta\right] K_{n}\left(k_{E} r\right),
\end{aligned}
$$

where the cosine modes have already been set to zero to ensure $\psi_{I}=-u a \sin \theta+\phi=0$. For $n=1$ the island boundary condition gives

$$
b_{W 1}=\frac{d_{W 1} Y_{1}\left(k_{W} a\right)\left(\Delta_{W}-\Delta_{E}\right)+\left(-u_{l}+\Delta_{E} u_{u}\right) a}{\left(\Delta_{E}-\Delta_{W}\right) J_{1}\left(k_{W} a\right)}
$$

and for $n=2$ it is

$$
b_{W 2}=-\frac{d_{W 2} Y_{2}\left(k_{W} a\right)}{J_{2}\left(k_{W} a\right)} .
$$

Forcing the upstream flow perturbations to be approximately zero $\left(\partial_{\theta} \phi(\theta=\pi)=0\right)$, we obtain (in the same manner as for the wavelike solutions)

$$
\begin{aligned}
& -d_{W 1}+2 b_{W 2}=0 \\
& -b_{W 1}-2 d_{W 2}=0 .
\end{aligned}
$$

Combining all of this gives

$$
\begin{aligned}
& b_{W 1}=\frac{\left(-u_{l}+\Delta_{E} u_{u}\right) a J_{2}\left(k_{W} a\right)}{\Upsilon_{W}\left(\Delta_{E}-\Delta_{W}\right)} \\
& b_{W 2}=\frac{1}{2} \frac{\left(-u_{l}+\Delta_{E} u_{u}\right) a J_{2}\left(k_{W} a\right)}{\Upsilon_{W}\left(\Delta_{E}-\Delta_{W}\right)} \\
& d_{W 1}=\frac{\left(-u_{l}+\Delta_{E} u_{u}\right) a Y_{2}\left(k_{W} a\right)}{\Upsilon_{W}\left(\Delta_{E}-\Delta_{W}\right)} \\
& d_{W 2}=-\frac{1}{2} \frac{\left(-u_{l}+\Delta_{E} u_{u}\right) a J_{2}\left(k_{W} a\right)}{\Upsilon_{W}\left(\Delta_{E}-\Delta_{W}\right)} \\
& d_{E 1}=\frac{-b_{W 1} J_{1}\left(k_{W} a\right)-d_{W 1} Y_{1}\left(k_{W} a\right)+u_{u} a}{K_{1}\left(k_{E} a\right)} \\
& d_{E 2}=\frac{-b_{W 2} J_{2}\left(k_{W} a\right)-d_{W 2} Y_{2}\left(k_{W} a\right)}{k_{2}\left(k_{E} a\right)}=0,
\end{aligned}
$$

with $\Upsilon_{W}=J_{1}\left(k_{W} a\right) J_{2}\left(k_{W} a\right)+Y_{1}\left(k_{W} a\right) Y_{2}\left(k_{W} a\right)$. 


\section{Appendix C: The supercritical transition}

The supercritical transition of the baroclinic-like mode may be illustrated by decomposing the modal solutions and background flows $(T$ and $C$ ) into strictly barotropic and baroclinic components, so that the full streamfunction decomposition is $\psi=\Psi_{b t}+\Psi_{b c}+\phi_{C b t}+$ $\phi_{C b c}+\phi_{T b t}+\phi_{T b c}$. The baroclinic and barotropic streamfunctions are defined

$$
\psi_{b t}=(1-\delta) \psi_{u}+\delta \psi_{l} \quad ; \quad \psi_{b c}=\sqrt{\delta(1-\delta)}\left(\psi_{u}-\psi_{l}\right),
$$

and other variables are decomposed similarly. The potential vorticity for the barotropic and baroclinic components is described by the following equations (Flierl 1978):

$$
\begin{array}{r}
\frac{\partial q_{b t}}{\partial t}+J\left(\psi_{b t}, q_{b t}\right)+J\left(\psi_{b c}, q_{b c}\right)=0, \\
\frac{\partial q_{b c}}{\partial t}+J\left(\psi_{b t}+\xi \psi_{b c}, q_{b c}\right)+J\left(\psi_{b c}, q_{b t}\right)=0
\end{array}
$$

where $\xi=-[\delta(1-\delta)]^{-1 / 2}$ and the baroclinic and barotropic potential vorticities are, respectively, $q_{b c}=\left(\nabla^{2}-1 / R_{D}^{2}\right) \psi_{b c}$ and $q_{b t}=\nabla^{2} \psi_{b t}+\beta y$. Writing $\psi_{b t}=-u_{b t} y+\phi_{b t}$ and $\psi_{b c}=-u_{b c} y+\phi_{b c}$, then linearizing Eq. (48) for steady solutions, the resulting coupled equations can be expressed in matrix form:

$$
\left[\begin{array}{cc}
u_{b t}-\frac{\beta}{k^{2}} & u_{b c} \frac{k^{2}}{k^{2}+1 / R_{D}^{2}} \\
u_{b c} \frac{k^{2}-1 / R_{D}^{2}}{k^{2}} & u_{b t}+\xi u_{b c}-\frac{\beta+1 / R_{D}^{2} \xi u_{b c}}{k^{2}+1 / R_{D}^{2}}
\end{array}\right]\left[\begin{array}{l}
q_{b t} \\
q_{b c}
\end{array}\right]=0
$$

where the wavenumber, $k$, is either $k_{T}$ or $k_{C}$, depending on the mode being decomposed. The phase speeds of the uncoupled barotropic and baroclinic components are given by the diagonal terms. As $k_{C}^{2} \rightarrow 0$, the mode becomes strictly baroclinic (indicated by the solid lines in panels $\mathrm{c}$ and $\mathrm{d}$ ), and the phase speed of the mode tends to its baroclinic limit:

$$
\begin{gathered}
\lim _{k_{C}^{2} \rightarrow 0}\left[c_{p}=u_{b t}+\xi u_{b c}-\frac{\beta+1 / R_{D}^{2} \xi u_{b c}}{k_{C}^{2}+1 / R_{D}^{2}}=0\right] \\
u_{b t}-R_{D}^{2} \beta=0 .
\end{gathered}
$$

The supercritical transition occurs where the baroclinic wave has infinite wavelength and its fastest phase speed, coinciding with the line $u_{b t}=R_{D}^{2} \beta$.

\section{REFERENCES}

Baines, P. G. and R. L. Hughes. 2016. Comments on "The Interaction of an Eastward-Flowing Current and an Island: Sub- and Supercritical Flow". J. Phys. Oceanogr., 46, 2263-2265. doi: 10.1175/JPOD-16-0016.1

Boyer, D. L. and P. A. Davies. 1982. Flow past a circular cylinder on a $\beta$-plane. Philos. Trans. A Math. Phys. Eng. Sci., 306(1496), 533-556. doi: 10.1098/rsta.1982.0094 
Cummins, P. F. and L. A. Mysak. 1988. A quasi-geostrophic circulation model of the Northeast Pacific. Part 1: a preliminary numerical experiment. J. Phys. Oceanogr., 18, 1261-1286. doi: 10.1175/1520-0485(1988)018<1261:AQGCMO>2.0.CO;2

Dong, C. and J. C. McWilliams. 2007. A numerical study of island wakes in the Southern California Bight. Cont. Shelf Res., 27(9), 1233-1248. doi: 10.1016/j.csr.2007.01.016

Flierl, G. R. 1978. Models of vertical structure and the calibration of two-layer models. Dyn. Atmos. Oceans, 2(4), 341-381. doi: 10.1016/0377-0265(78)90002-7

Gerrard, J. 1978. The wakes of cylindrical bluff bodies at low Reynolds number. Phil. Trans. R. Soc. Lond. A, 288(1354), 351-382. doi: 10.1098/rsta.1978.0020

Hallberg, R. 1997. Stable split time stepping schemes for large-scale ocean modeling. J. Comput. Phys., 135(1), 54-65. doi: 10.1006/jcph.1997.5734

McCartney, M. S. 1975. Inertial Taylor columns on a beta plane. J. Fluid Mech., 68(1), 71-95.

Merkine, L.-O. 1980. Flow separation on a $\beta$-plane. J. Fluid Mech., 99(02), 399-409.

Miles, J. 1968. Lee waves in a stratified flow Part 1. Thin barrier. J. Fluid Mech., 32(3), 549-567.

Pedlosky, J. and M. A. Spall. 2015. The interaction of an eastward-flowing current and an island: suband supercritical flow. J. Phys. Oceanogr., 45(11), 2806-2819. doi: 10.1175/JPO-D-15-0061.1

Rhines, P. B. 2007. Jets and orography: idealized experiments with tip jets and Lighthill blocking. J. Atmos. Sci., 64(10), 3627-3639.

Salmon, R. 1998. Lectures on geophysical fluid dynamics. Oxford: Oxford University Press.

Schönau, M. C. and D. L. Rudnick. 2015. Glider observations of the North Equatorial Current in the western tropical Pacific. J. Geophys. Res.: Oceans, 120(5), 3586-3605. doi: 10.1002/ 2014JC010595

Tansley, C. E. and D. P. Marshall. 2001. Flow past a cylinder on a beta plane, with application to Gulf Stream separation and the Antarctic Circumpolar Current. J. Phys. Oceanogr., 31, 3274-3283. doi: 10.1175/1520-0485(2001)031<3274:FPACOA >2.0.CO;2

Tulloch, R., J. Marshall, C. Hill, and K. S. Smith. 2011. Scales, growth rates, and spectral fluxes of baroclinic instability in the ocean. J. Phys. Oceanogr., 41(6), 1057-1076. doi: 10.1175/2011JPO4404.1

Van Dyke, M. 1982. An album of fluid motion. Stanford: Parabolic Press.

White, W. B. 1971. A Rossby wake due to an island in an eastward current. J. Phys. Oceanogr., 1, 161-168.

Received: 1 February 2018; revised: 20 June 2018. 